\title{
Recent progress in periodic patterning fabricated by self-assembly of colloidal spheres for optical applications
}

\author{
Jing Liu ${ }^{1 \dagger}$, Xingang Zhang ${ }^{3 \dagger}$, Wenqing $\mathrm{Li}^{1}$, Changzhong Jiang ${ }^{1}$, Ziyu Wang ${ }^{2^{*}}$ and Xiangheng Xiao ${ }^{1,4^{*}}$
}

\begin{abstract}
Colloidal crystals are periodically ordered arrays of monodisperse colloidal particles which represent a new class of self-assembled materials showing potential applications in many fields. Two-dimensional graphic nanostructures based on colloidal crystals have inherent periodicity from tens of nanometers to several micrometers, which gives them rich and interesting optical properties. This article presents a comprehensive review about the current research activities on the self-assembly of colloidal spheres which is an effective strategy for fabrication of various hierarchical and ordered nanostructures, with particular attention paid to the unique properties and applications of the colloidal crystal-based nanostructures. Three main aspects are elaborated: a) controllable self-assembly of colloidal crystals; b) the functions of the obtained colloidal spheres acting as the patterned mask for successive construction of numerous nanostructures; c) the novel properties and promising optical applications of the patterned nanostructures in various domains, such as plasmonic-related fields, antireflection, photonic crystals, photocatalysis and electronic devices. After that, the current challenges and future perspectives in this area are provided. This review aims to inspire more ingenious designs and exciting research for manufacturing nanostructures utilizing colloidal self-assembly.
\end{abstract}

Keywords: colloidal crystals, self-assembly, patterned nanostructures, plasmonic, antireflection, photonic crystals

\section{INTRODUCTION}

Nanostructures are a new system based on nanoscale material units that are constructed or built according to certain rules, including nanoparticles, stable clusters, or artificial super-atoms, nanotubes [1], nanorods [2], nanowires [3,4], and nanometer-sized pores [5]. Surfaces with ordered nanostructures have attracted intensive interest due to their distinctive surface feature, unique-dependent properties and potential applications in a variety of important technological fields [6]. Therefore, it is necessary to develop effective nano-patterning technique to achieve high-throughput and low-cost production of large-area periodic nanostructures with adjustable structural parameters. Nanostructures can be fabricated by using a number of advanced methods, such as photolithography, soft lithography, electron beam lithography, and nanoimprinting [7-10], however, usually suffer from high cost and low throughput, or are limited by the prerequisite preparation of appropriate templates. Therefore, further development of large-scale practical fabrications with less demand on instrumentation still requires great ingenuity $[11,12]$. In this regard, the surface stencil method using a self-assembly process to produce templates is highly efficient in producing periodic nanostructures over a large area [13-15]. In particular, the manufacturing and assembly methods based on singlelayer colloidal crystal templates, through the early modification of colloids and the subsequent photolithographic

\footnotetext{
${ }^{1}$ School of Physics and Technology, Key Laboratory of Artificial Micro- and Nano-structures of Ministry of Education, Hubei Nuclear Solid Physics Key Laboratory, Wuhan University, Wuhan 430072, China

${ }^{2}$ Institute of Technological Sciences, Wuhan University, Wuhan 430072, China

${ }^{3}$ Synergetic Innovation Center for Quantum Effects and Application, Key Laboratory of Low Dimensional Quantum Structures and Quantum Control, School of Physics and Electronics, Hunan Normal University, Changsha 410081, China

${ }^{4} \mathrm{Su}$ Zhou Institute of Wuhan University, Suzhou 215123, China

${ }^{\dagger}$ These authors contributed equally to this work.

* Corresponding authors (emails: zywang@whu.edu.cn (Wang Z); xxh@whu.edu.cn (Xiao X))
} 
processing, have proven to be superior due to their unique advantages such as low loss and easily controlled chemical composition and structural parameters.

Generally speaking, colloidal lithography uses the ordered stacking of spherical and self-assembled colloidal crystals of a single nanosphere to produce an ordered array of nanostructures with the same lattice structure and period as two-dimensional (2D) colloidal crystals. The feature size can easily shrink by decreasing the microsphere diameter in the colloidal mask. The feature shape can be diversified by varying the crystal structure of the colloidal mask, etching the mask, altering the incidence angle of the vapor beam, and stepwise manipulating of the mask registry. These nanostructure arrays have broad application prospects, such as photonic crystals, catalysis, templates for fabricating various structures [16-20]. With the development of colloid science, monodisperse colloidal spheres, such as polystyrene or silica, with narrow particle size distribution and good phase stability were synthesized by emulsion, suspension, and various methods [21]. The size of the colloidal spheres can be accurately adjusted within a range from a few micrometers to tens of nanometers, which is equivalent to or better than the resolution limit of traditional patterning techniques [22]. Under appropriate conditions, such colloidal spheres can self-assemble into periodically ordered colloidal crystals [23-25]. Colloidal crystals have attracted much attention because of their important application prospects in photonic crystals, inverse opal manufacturing templates, and material crystallization process research. In 1981, scientists first proposed a photolithography method using self-assembled polystyrene colloidal spheres as a mask [26]. Since then, many efforts have been made to arrange colloidal nanospheres into complex structures [27-29]. Surface features of ordered nanostructures based on colloidal lithography were firstly derived from the formation of monolayer colloidal spheres, followed by modification and nanofabrication [30]. The preparation and further modification of the colloidal mask is the premise of colloidal lithography, and it is of great significance to obtain the ideal structure and performance.

In this review, we present a systematic overview of the recent research activities based on ordered nano-structure arrays, from controllable colloidal self-assembly, especially polystyrene microspheres, and their modifications combined with nano-fabrication techniques to the optical applications in various domains. First, we elaborate on the preparation of nanostructures from the perspective of functional materials and substrates, and fabricate a large- area, highly ordered periodic hexagonal close-packed structure using self-assembly technology. Then, various layered periodic pattern structures are formed by nanosphere lithography, controllable deposition technology, and precursor solution assistance. After the introduction in this section, focus will be shifted to summarize their wide applications, especially in optical and photonic related topics including anti-reflectance, photonic crystals, photo-electrochemistry, and surface-enhanced Raman spectroscopy (SERS) substrates. The recent advances in ordered nanostructures and the novel properties and emerging applications of nanostructures based on the introduced functional materials in various fields are summarized next. Finally, conclusions are drawn with a short outlook on the future development in this area.

\section{CONTROLLABLE SELF-ASSEMBLY OF COLLOIDAL CRYSTALS}

In colloidal technology, producing a monolayer colloidal template through the self-assembly approach has been verified to be an effective and versatile route toward functional periodic nanostructures. However, it is still a difficult task to achieve the large-scale, controllable and uniform self-assembly colloidal spheres in a quick and cost-effective way. Once the high-quality monolayer colloidal crystals (MCCs) are obtained, they can act as the mask or template [31,32]. The assembly of colloidal crystals and their further modifications are more attractive in generating new nanostructures with desired properties. At present, there is no mature view on the division of nanostructure assembly systems, but only construction based on nanostructure systems, which roughly fall into two categories: one is the artificial nanostructure assembly system, and the other is the nanostructure self-assembled system and the molecular selfassembled system. Nano- and micro-scale monodisperse spheres can spontaneously form hexagonal close-packed MCCs when a thermodynamically stable suspension is dried under certain free volume constraints. Colloidal nanospheres dispersed in various solvents are influenced by interactions including van der Waals forces, coulombic repulsions and steric repulsions. Self-assembly of the colloidal nanospheres is governed by the balance among these interactions. Self-assembly conditions and parameters, such as temperature, air pressure, humidity and solvent evaporation rate are crucial to influencing the ordering and improving the quality of colloidal crystals. Between the solid spheres in a colloidal crystal are interstices that are arranged into an ordered structure, inverted to the ordered structure of the solid spheres. So far, 
a host of colloidal crystallization strategies have been successfully established, which belong to one of four different schemes, namely drop-coating, dip-coating, spin-coating, and self-assembly at air-liquid interface [33-35].

\section{Self-assembly of monolayer colloidal crystals}

Self-assembled 2D colloidal crystals are single-layer arrays composed of colloidal microspheres or nanospheres. The conditions and parameters of self-assembly, such as temperature, humidity and solvent, are the key controlling the morphologies and qualities of colloidal crystals. In 1992, Bulgarian Sofia scientists [36] first observed the 2D dynamics of polystyrene microspheres with an optical microscope. They found that when the thickness of the solvent layer was close to the diameter of the nanospheres, the nanoparticles began to sort regularly. Based on this phenomenon, they developed a dip coating process to form tightly packed hexagonal single-layer colloidal crystals. However, due to the convective transport of particles to the ordered region, the later growth of the crystal could proceed. Experiments were carried out under the control of crystal growth, and an ordered monolayer or a domain composed of multiple layers was obtained [37-39]. Recently, Gao and co-workers [40] presented a new patterning ultrahigh throughput micropropulsive injection process, which can directly produce periodic MCCs with area of several square meters, as illustrated in Fig. 1a. The corresponding scanning electron microscopy (SEM) images and some specific pictures can be seen from Fig. 1b-e. At the same time, based on this efficient and fast way, Retsch and co-workers [41] presented an equally rapid method for the fabrication of close-packed colloidal crystals with large domain sizes through floating and redeposition of colloidal monolayers at the air/water interface, as illustrated in Fig. 1f, which is the generic approach to produce MCC. At first, the deposition of a colloidal dispersion drops on the initial solid substrate. Then after drying of the dispersion, the particles are floated at the air and water interface through slow immersion of the parent substrate under a shallow angle. The polystyrene particles depart from the substrate at the contact line, self-assemble into a dense monolayer, and remain afloat at the air/water interface (Fig. 1g) [42]. In order to reduce the defect concentration of the prepared single-layer colloidal crystals, two different ultrasonic annealing techniques and special-frequency barrier swing techniques can be used, as shown in Fig. 1g. The ultrasonic annealing device consists of an ultrasonic generator and a transducer, and an oscillation of 1.2 to $1.5 \mathrm{MHz}$

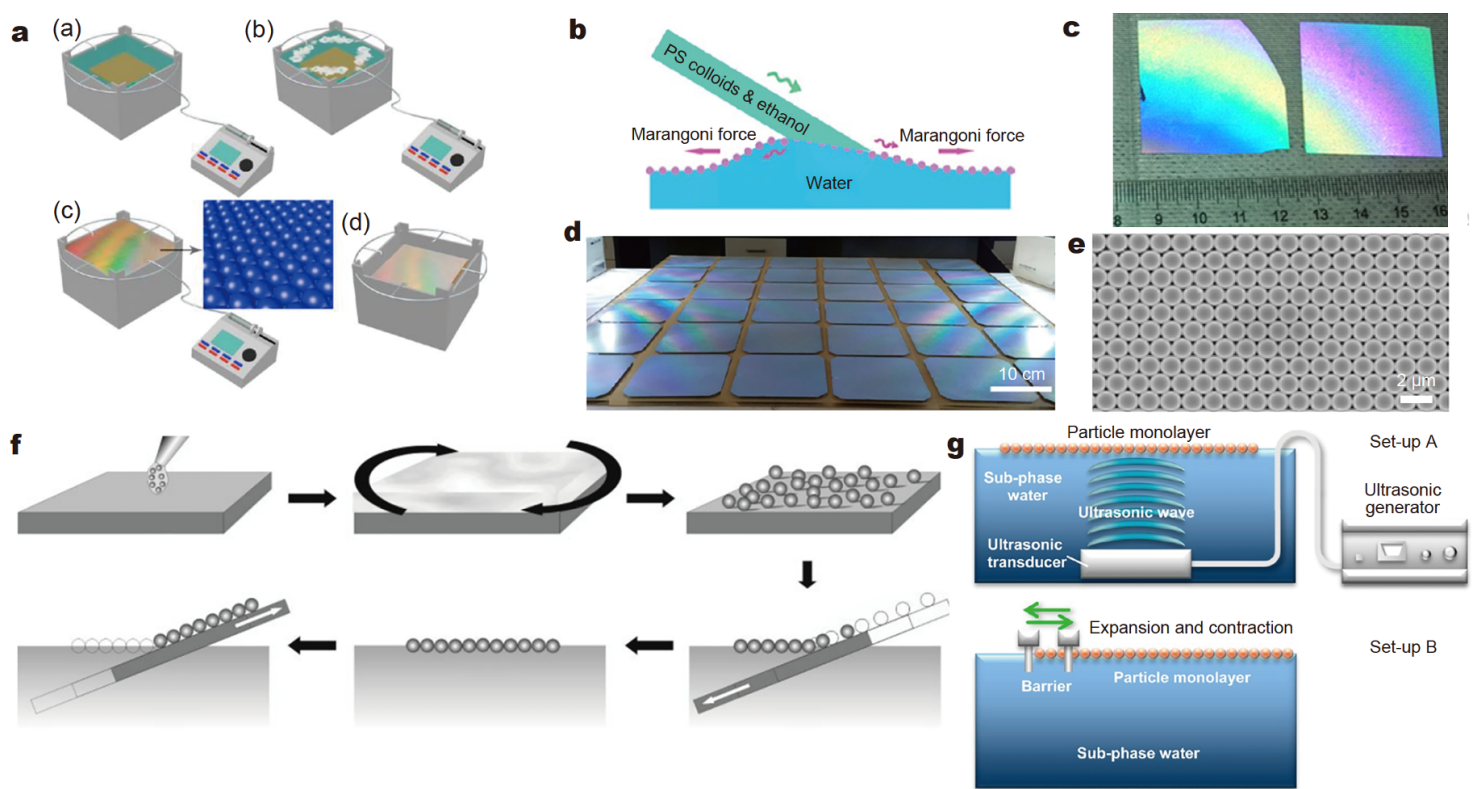

Figure 1 Monolayer colloidal crystal based on self-assembly at air-liquid interface. (a) Schematics of the micro propulsive injection systems and the formation processes for large-area polystyrene nanosphere arrays. (b-e) Schematic and photograph of the dynamic and equilibrium behaviors near the interface between the injector tip and the water surface. Reproduced with permission from Ref. [40]. Copyright 2015, American Chemical Society. (f) Monolayer formation at the air/water interface from a spin coated parent substrate by the self-assembly method. Reproduced with permission from Ref. [41]. Copyright 2009, Wiley-VCH. (g) Schematic illustrations of the setup of Langmuir-Blodgett trough ultrasonic irradiation and barrier-sway process to the particle monolayer. Reproduced with permission from Ref. [42]. Copyright 2015, American Chemical Society. 
irradiates from the transducer head to the water surface at $20^{\circ} \mathrm{C}$ for $30 \mathrm{~min}$. At the same time, the silicone ball drops and then spreads on the water surface (set-up A). By contrast, a physical treatment process called barrier-sway causes the silica spheres to sway forward and backward alternately on the water surface through a maneuvering barrier slot (set-up B). The floating layer of colloidal particles can then be picked up by a second substrate, which is called the receiving substrate. To achieve this, the receiving substrate is immersed in water and slowly retracted at a shallower angle, just the opposite of the immersion process. Finally, the receiving substrate is held at a fixed angle until all the liquid has evaporated. In addition, there are many large-area MCCs synthesized from other materials, such as polymethyl methacrylate (PMMA), Janus particles, and flexible materials such as microgels [43-45].

\section{Binary monolayer colloidal crystals}

Monolayer-nanoparticle array obtained from the techniques mentioned above can be used as the second template or mask for the following process. Binary colloidal crystals are colloidal crystals assembled from two kinds of monodisperse colloidal particles with different particle sizes. Relative to the finite crystal structure assembled using single-size colloidal spheres, binary colloidal crystals exhibit rich crystal symmetry and different stoichiometry in size ratio and concentration. The effect of small spheres on the final dense structure of large spheres may be very limited. Therefore, periodic ordered binary colloidal crystal patterns can be designed by selecting large colloidal spheres of the required size.

As a new method for preparing binary MCCs, selfassembly can be divided into the co-assembly method and step-by-step assembly method. Compared with the finite crystal structure assembled using single-sized colloidal spheres, they show a rich symmetrical arrangements of crystals and different stoichiometries, depending on the size ratio and concentration [46-48]. After oxygen plasma treatment, various controllable morphologies of nanoparticles can be achieved with the etched spheres acting as a template during the metal deposition. More recently, monolayer binary colloidal crystal patterns were fabricated by the co-assembly (Fig. 2a) [49]. At first, a suspension was spread at the air-water interface through a sloping glass slide; colloidal nanospheres tended to move toward the gas-liquid interface after surface modification to enhance the hydrophobicity of the surface, then sodium dodecyl sulfonate (SDS) was added to push and consolidate the close-packed monolayer; the monolayer

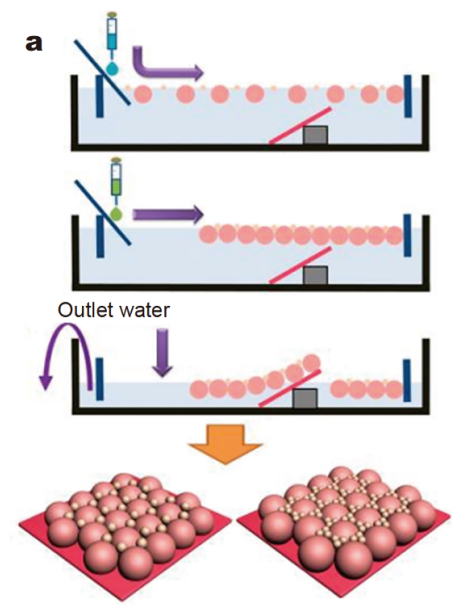

b
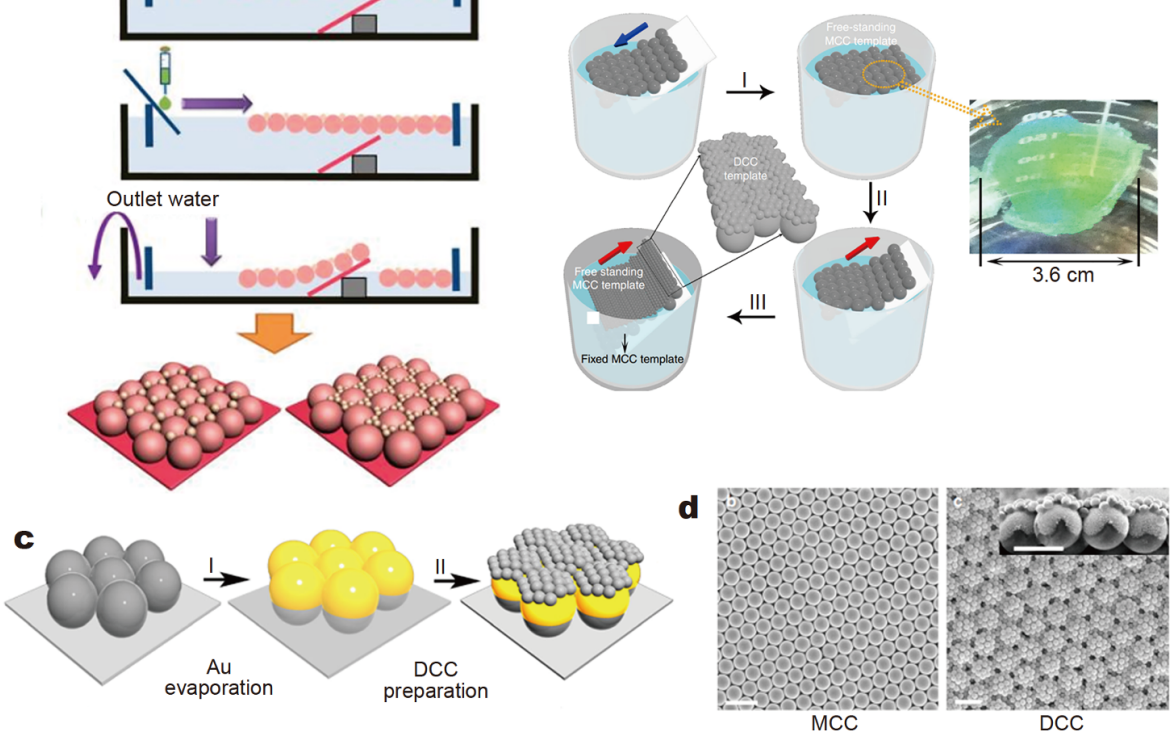

Figure 2 Binary monolayer colloidal crystal self-assembly system. (a) Illustration of the procedure for the fabrication of monolayer binary colloidal crystals and their transfers to substrate. Reproduced with permission from Ref. [49]. Copyright 2014, American Chemical Society. (b) Fabrication process of DCC template. (c) Corresponding schematic of (b). (d) SEM images of MCC and DCC templates, respectively (scale bars: $2 \mu \mathrm{m}$ ). Reproduced with permission from Ref. [51]. Copyright 2017, Springer Nature Publishing. 
was then transferred to a specified substrate surface by lowering the water slowly.

Based on stepwise assembly, our group [50] have developed a layer-by-layer growth process and prepared large-area 3D hierarchical sunflower-like nanoarrays. On the basis of the complex binary-colloidal crystal patterns and subsequent oxygen plasma processing, we point to a new method of designing the dimension and separation of nontraditional evaporated structures. Similar work was reported by Yang and co-workers [51]. They synthesized coatings by first preparing a highly ordered double-layer colloidal crystal (DCC) template and then conducting site-specific electrodeposition on the template. The DCC template was prepared through a layer-by-layer stacking process. Some stepwise-assembly methods have been developed to fabricate monolayer binary-colloidal structure patterns, as shown in Fig. 2b; the binary colloidal crystal template was prepared through a layer-by-layer stacking. First, a monolayer colloidal crystal template composed of polystyrene spheres was prepared through spin-coating, and then transferred onto the water/air interface (Process I in Fig. 2b). An arbitrary substrate was used to pick up the free-standing MCC template from underneath (Process II in Fig. 2b). The substrate could be in the form of a smooth, flexible, curved, or roughened surface. As illustrated in Fig. 2c, a binary colloidal crystal pattern was obtained after another layer of the colloidal crystal template was transferred onto the pre-fabricated colloidal crystal template. Fig. $2 \mathrm{~d}$ shows the corresponding SEM images.

\section{COLLOIDAL CRYSTAL-ASSISTED CONSTRUCTION OF VARIOUS HIERARCHICAL NANOSTRUCTURE ARRAYS}

Once large-scale, high-quality monomolecular colloidal crystals are obtained, various functional materials are used to make ordered nanostructures with required properties. In addition to the single-layer colloidal crystals obtained directly by self-assembly, the basic arrays of nano-objects such as dots, holes, and meshes can also be used as templates for subsequent processes. In addition, controlled physical deposition paths including nanoetching, vapor deposition and catalytic growth techniques, as well as chemical deposition paths including solution interface assembly and reaction techniques, and liquid precursor-filled template sphere deposition have been used to fabricate nanostructures. Due to their wellcontrolled size, morphology and organization, as well as the inherent properties of the materials, these nano- structures have great potential in many important areas.

\section{Controllable etching assisted by monolayer colloidal crystals}

Monolayer colloidal crystal-assisted etching is a simple and straightforward method for preparing various $2 \mathrm{D}$ and even 3D patterned nanostructures. Compared with traditional photolithography, nanosphere marking technique can significantly reduce the cost and complexity of manufacturing. Therefore, in order not to be restricted by expensive and complicated equipment, periodic nanostructure preparation can be widely applied on many manufacturing platforms [52]. In this regard, the combination of colloid-based self-assembly lithography methods and other nanofabrication technologies can provide a novel alternative and interesting strategy to create periodic nanostructures.

In practice, the properties of materials are highly dependent on the shape and size of the nanostructures. Therefore, it is necessary to prepare various nano-shaped structures that meet the requirements [53]. The limited regulation of nanostructure arrays is a significant disadvantage of colloidal spheres. To overcome this shortcoming, deep reactive ion etching [54], ion milling [55] or annealing [56] provides an effective method for preparing non-spherical nanostructure arrays $[57,58]$. As shown in Fig. 3, our group [59] prepared well-organized inverted cone nanostructures on multilayer polystyrene colloidal crystals by using plasma etching, with the top layer as a shadow mask. First, a small polystyrene single-layer colloidal crystal sphere template prepared through a selfassembly process was superimposed on a template composed of large polystyrene spheres to obtain a doublelayered colloidal crystal structure. The small polystyrene spheres were closely packed in hexagons on the large polystyrene spheres, and acted as sacrificial dynamic protection templates during the plasma etching process to form ordered nano-cones on the large polystyrene spheres. The obtained pattern and particle shape could
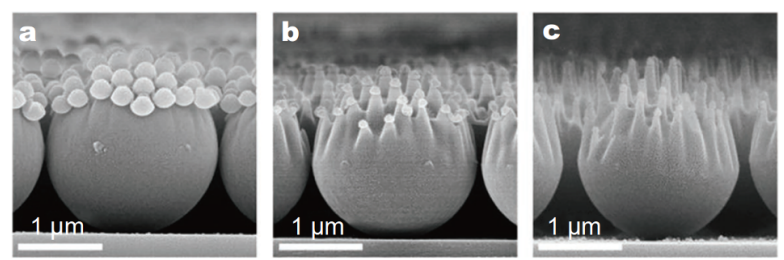

Figure 3 SEM images of the double-layer colloidal sphere template after plasma etching for (a) $15 \mathrm{~min}$, (b) $45 \mathrm{~min}$, and (c) $70 \mathrm{~min}$, respectively. Reproduced with permission from Ref. [59]. Copyright 2019, Wiley$\mathrm{VCH}$. 
continue to be used as a secondary template or mask for subsequent processes such as chemical vapor deposition and metal sputtering and the shape of nano-array depended on the number of colloidal crystal layers and plasma etching conditions $[60,61]$.

Once obtained, periodic nanostructures can serve as a mask for metal deposition. Due to the difficulty of plate making with metal catalytic lattice, the traditional plate making method is difficult to realize. With the help of polystyrene microspheres, metal-assisted chemical etching can be used to generate nanoarrays of other materials in various shapes [62,63]. Zhu and his colleagues [64] combined colloidal photolithography with metal-catalyzed etching to produce silicon nanowire arrays with controlled diameter, length, and density. This is a mature chemical etching scheme that can obtain highly anisotropic nanostructures under the action of specific metal catalysts. Specifically, the polystyrene sphere monolayer was subjected to oxygen plasma in order to reduce the diameter of polystyrene spheres, and then $\mathrm{Au}$ film was deposited onto the substrate via thermal evaporation [65]. Furthermore, nanospheres can be used as a resist and can be formed in parallel in each processing step. After the reactive ion etching, the polystyrene spheres were significantly reduced and the arrangement was no longer tight. This nanofabrication technology uses selected and repeatedly etched polymer nanospheres. Fig. $4 \mathrm{a}$ is based on photolithography of nano-microspheres combined with metal-assisted chemical etching technology to prepare a highly ordered hollow structure silicon nanorod array [66]. The corresponding SEM images can be seen from Fig. 4b. First, a single layer of polystyrene microsphere mask was formed on the surface of the Si substrate for repeated etching. The diameter of the polystyrene nanospheres was reduced by oxygen plasma etching to determine the outer diameter of the silicon nanotubes. In the next step, oxygen plasma etching was used again to reduce the size of the polystyrene nanospheres on top of the pillars. Similarly, smaller nanospheres would be used as a mask for subsequent deep reactive ion etching techniques. Finally, a completed Si nanotube array was obtained after being subjected to a chemical etching process. The corresponding relationship between oxygen plasma reactive ion etching time and size of polystyrene nanoparticles can be seen from Fig. 4c. By selecting the proper original nanosphere size, the pitch of the final silicon nanotube array could be effectively controlled. This method is suitable for constructing $3 \mathrm{D}$ periodic layered hybrid nanostructures [67]. The height and diameter of each layer of each structure can be precisely

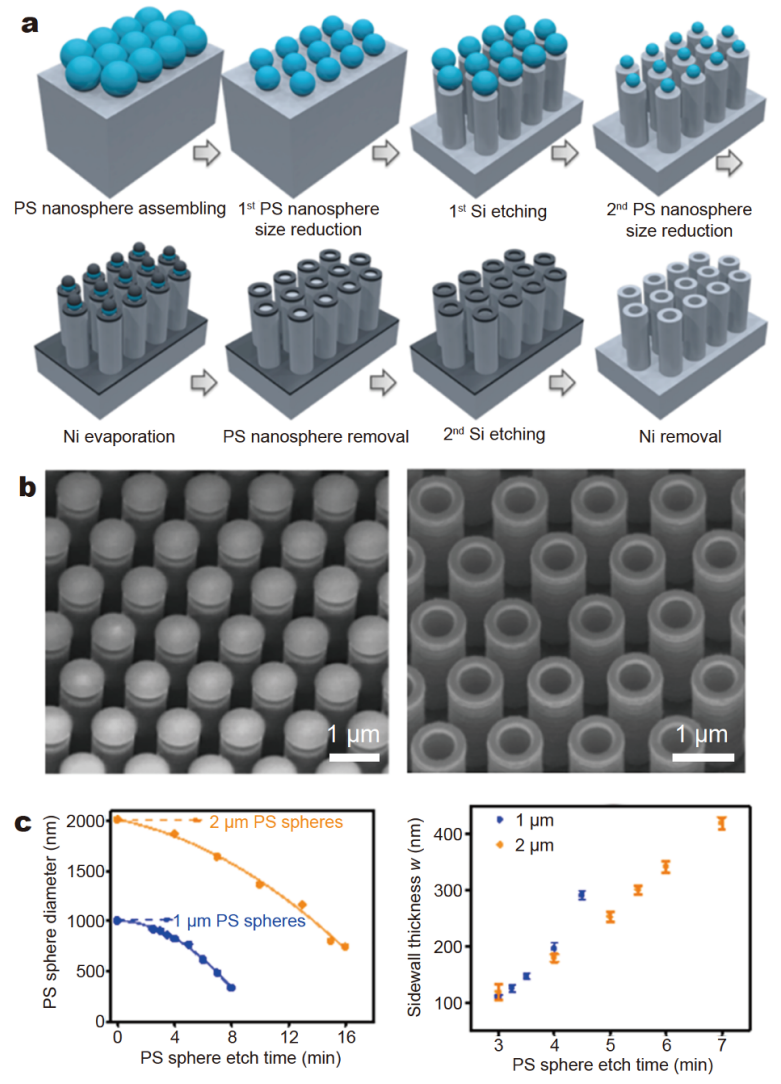

Figure 4 (a) Schematic illustration of the fabrication steps. (b) SEM images of the representative periodic Si nanotube arrays with different parameters in $\mathrm{nm}$. (c) Corresponding relationship between oxygen plasma reactive ion etching time and size of polystyrene nanoparticles. Reproduced with permission from Ref. [66]. Copyright 2017, American Chemical Society.

configured, and the ring has adjustable optical characteristics on various substrates.

\section{Controllable deposition assisted by monolayer colloidal crystals}

In addition to the combination of colloidal lithography and nanofabrication technology, single-layer colloidal crystal-assisted deposition is also a very powerful technology for manufacturing various nanostructured functional materials, providing various places for target materials to be deposited or grown. The material can be selectively deposited on the surface of the colloidal nanospheres, the gaps between adjacent nanospheres, or on the exposed substrate. By selecting a suitable deposition method, adjusting the deposition conditions, using polystyrene spheres as a mask, and combining colloidal crystal-assisted deposition techniques, various $2 \mathrm{D}$ patterned nanostructures that rely on pattern features can be 
prepared.

It is worth noting that because of its simplicity and flexibility in morphology control, the hydrothermal method has become one of the most popular methods for nanomaterial preparation [68,69]. Monolithic colloidal crystal nanolithography can be combined with hydrothermal methods to utilize different material properties to generate many interesting hierarchical structures and periodic nanoarrays [70-73]. Fig. 5a shows a $\mathrm{ZnO}$ nanowire array synthesized on indium tin oxid (ITO) glass substrate with a hydrothermal method combined with a nanosphere lithography scheme [74]. Specifically, $\mathrm{Zn}$ precursor colloidal suspension was spin-coated on an ITO glass substrate, and a single layer of polystyrene spheres was prepared on the top of the substrate, after that, an $\mathrm{Al}$ film with a thickness of $50 \mathrm{~nm}$ was deposited using electron beam. After the polystyrene sphere single layer was removed, a periodic $\mathrm{Al}$ nanopore array was obtained, and then a layered periodic $\mathrm{ZnO}$ nanowire was prepared on the substrate using a hydrothermal method. To pursue smaller nanogaps, Zhu and co-workers [75] demonstrated a simple and economical method that used a binary template-assisted electrodeposition method to vertically grow large-area ordered silver nanorod bundles on the $\mathrm{Au} / \mathrm{Cu}$ substrate, as shown in Fig. 5b. Firstly, a layer of polystyrene spheres was densely self-assembled on the surface of the ordered porous alumina (AAO) film, and then the diameter of the polystyrene spheres was reduced by plasma etching. After that, a $200 \mathrm{~nm}$-thick $\mathrm{Au}$ layer was sputtered onto the top surface of the polystyrene nanospheres and then the polystyrene microspheres were removed. Finally, silver nanorods were obtained by the electrodeposition method, and the AAO film was completely etched in $\mathrm{NaOH}$ solution. Due to the capillary force, the silver nanorods are close to each other, forming a large-area ordered array of silver nanorod bundles. The advantage of this manufacturing method is that a uniform and ordered array of Ag-nanowire bundles can be achieved using an ordered porous anode AAO film combined with a polystyrene sphere template. The resulting structure is highly adjustable and reproducible.

Physical sputtering deposition has also been used to create ordered metallic nanostructures. Wang and coworkers [76] utilized physical sputtering deposition to deposit a thin $\mathrm{TiO}_{2}$ film on the surface of self-assembled colloidal nanospheres. Similar colloidal-crystal-assisted deposition strategies have also been used for fabrication of ordered arrays of $\mathrm{Cu}$ nanoparticles [77]. $\mathrm{Li}$ and coworkers [78] fabricated periodic hexagonal spherical nanoparticle arrays with $10 \mathrm{~nm}$ gaps using MCC-induced

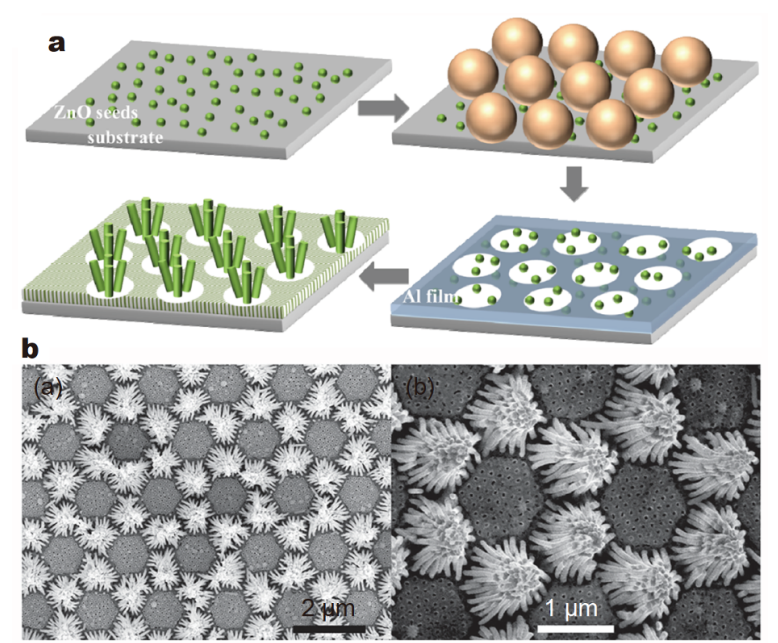

Figure 5 (a) Schematic illustration of $\mathrm{ZnO}$ nanowire/nanosheet synthesis. Reproduced with permission from Ref. [74]. Copyright 2015, The Optical Society. (b) SEM images of the as-prepared Ag-nanorod bundle arrays. Reproduced with permission from Ref. [75]. Copyright 2016, Wiley-VCH.

solution dipping and further physical sputtering deposition. A low detection limit of $10^{-12} \mathrm{~mol} \mathrm{~L}^{-1}$ rhodamine $6 \mathrm{G}$ can be achieved after the modification of nanoparticle arrays with a low-surface-free-energy material. Periodic spherical nanoparticle arrays were fabricated through sputtering deposition of regular network-structured arrays with prism-like protrusions among three neighboring holes templated by colloidal monolayer, as illustrated in Fig. 6. In addition to physical deposition, 2D colloidal crystals are also used as templates for electrochemical polymerization to prepare ordered conductive polymers [79].

\section{Controllable framework assisted by colloidal crystal}

Porous materials have attracted considerable scientific attention due to their widespread applications. The ordered structure with a large pore size can be synthesized by filling the gap between the template spheres with a liquid precursor $[80,81]$, and then removing the template spheres after the reaction is initiated. The gap-filled liquid precursor may be a UV- or thermally-initiated prepolymer, an organic monomer to which an initiator is added, a sol-gel precursor of an inorganic ceramic material, an inorganic salt solution, or a colloidal state.

Polystyrenes are employed as hard templates to generate macropores. Ga-doped hierarchical porous $\mathrm{TiO}_{2}$ $\mathrm{SiO}_{2}$ microarray with an anatase framework is fabricated for the nonoxidative coupling of $\mathrm{CH}_{4}$, which exhibits a high conversion rate with $90 \%$ selectivity (Fig. 7a) [82]. 

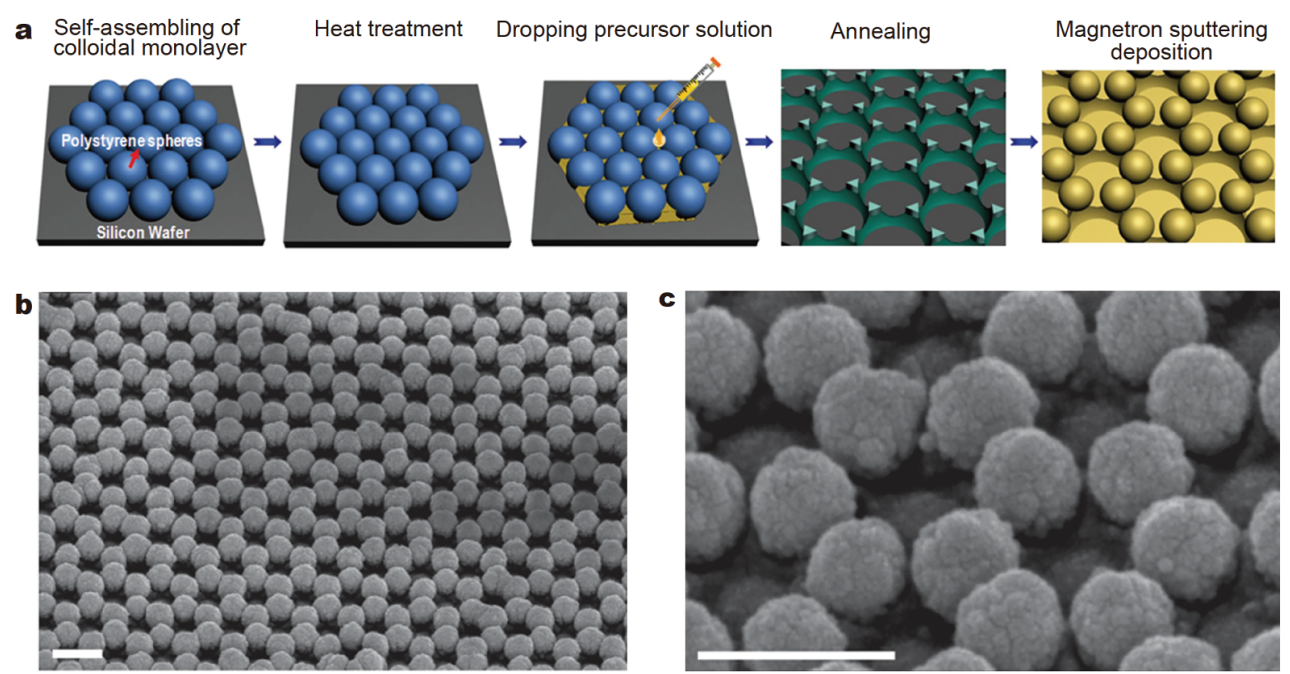

Figure 6 (a) Fabrication strategy for periodic spherical nanoparticle arrays. After magnetron sputtering deposition at $50 \mathrm{~W}$ for a certain time, hexagonal periodic spherical nanoparticle arrays were formed. (b) SEM images of periodic spherical nanoparticle arrays using regular networkstructured arrays as sputtering deposition template. (c) Expanded image of (b). Scale bars: $500 \mathrm{~nm}$. Reproduced with permission from Ref. [78]. Copyright 2015, Wiley-VCH.

a
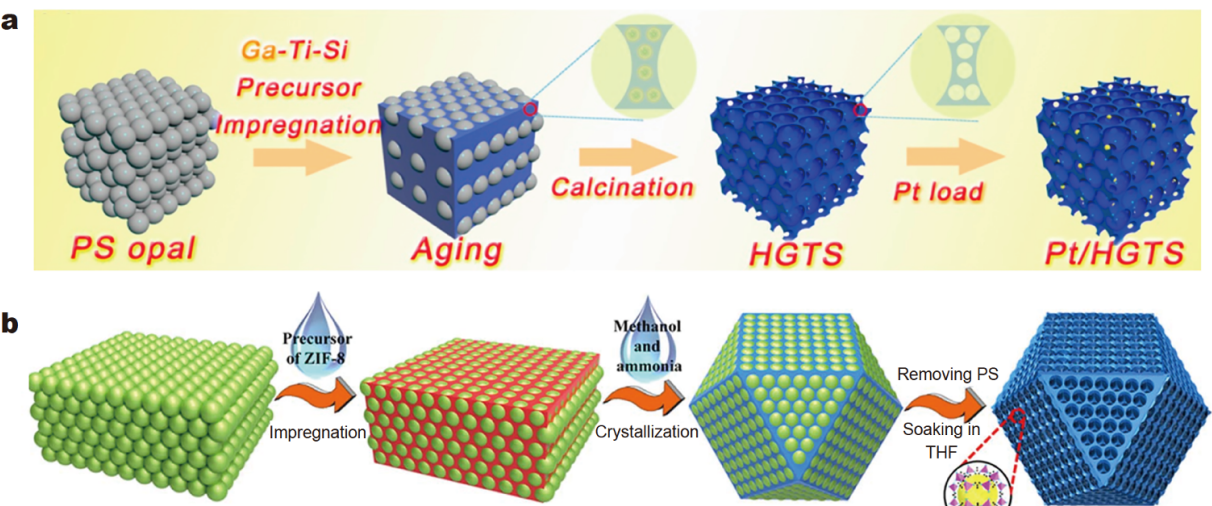

PS
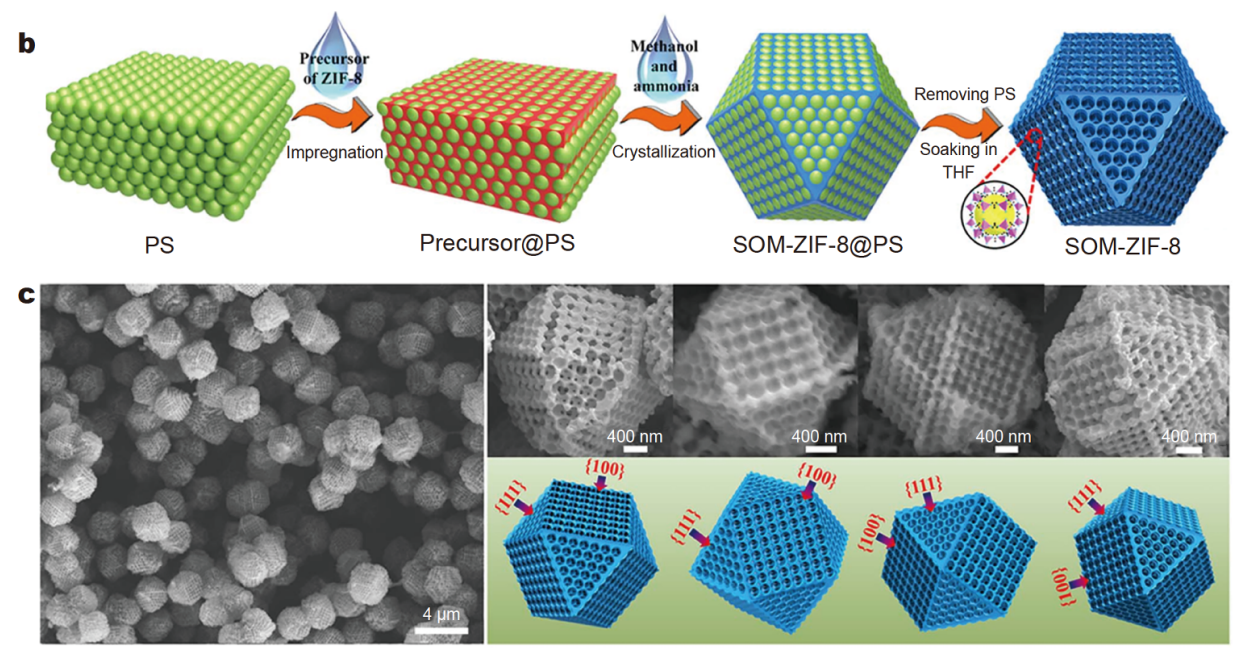

Figure 7 Colloidal crystal assist in forming controllable frameworks. (a) Schematic illustration of the synthesis process for Pt-hierarchical macromesoporous structure (Pt/HGTS). Reproduced with permission from Ref. [82]. Copyright 2019, American Chemical Society. (b) Schematic diagram of the fabrication method. (c) In situ nano-casting synthesis of single-crystal ordered macropore (SOM-ZIF-8) on the external surface of the polystyrene sphere template and its structure confirmation. Reproduced with permission from Ref. [83]. Copyright 2017, AAAS.

The polystyrene opals were obtained by drying the polystyrene emulsion in a $343 \mathrm{~K}$ oven for 3 days. As illustrated in Fig. 7b, Shen and co-workers [83] constructed highly oriented and ordered macropores within metalorganic framework (MOF) single crystals, opening up the area of $3 \mathrm{D}$-ordered macro-microporous materials in 
single-crystalline form. Their methodology relies on the strong shaping effects of a polystyrene nanosphere monolith template and a double-solvent-induced heterogeneous nucleation approach. This process synergistically enables MOFs to grow in situ in ordered voids, forming single crystals with oriented, ordered macroscopic microporous structures. This structure has exhibited superior structural stability and improved recyclability as shown in Fig. 7c. All above results can clearly reveal the versatility and the reproducibility of this unique fabrication framework.

\section{PROPERTIES AND APPLICATIONS OF COLLOIDAL CRYSTAL-BASED NANOSTRUCTURES}

Ordered nanomaterials play an important role in electronics, optics, magnetism, environmental detection, and energy conversion, due to their special properties, such as simple structure, low cost, and controllable morphology $[84,85]$. In this part, we will summarize a range of applications based on self-assembled colloidal spheres, with an emphasis on the optical and photonic-related fields including noble metal nanostructures for SERS, photoelectrochemistry, electronic device applications, and biomimetic antireflective coatings.

\section{Noble metal nanostructures for SERS}

The noble metal nanostructures have significantly superior surface plasmon resonance absorption, which is caused by the collective excitation of electrons caused by incident electromagnetic radiation near the metal-dielectric interface and coherent oscillation along the surface [86-90]. With the continuous deepening of the research on surface plasmon and the constant advancement of nano-processing technology, we can accommodate different applications through good control of material morphology [91]. Local surface plasmons exist when the collective resonance is localized within the near surface region of the metal objects [92]. For a metal nanostructure, when its size is equal to the surface depth, the power plant of incident light can penetrate the metal and polarize the electrons $[93,94]$. Therefore, plasmons in nanostructures can be regarded as spring resonators caused by light wave energy resonance [95-97]. In general, the morphology and interparticle distance of precious metal nanostructures are very sensitive and can be used for light-induced hot carrier transfer and to enhance the Raman spectrum signal of the substrate.

Metal nanostructures have sharp tips or are separated by nano-gaps, limiting the strong electromagnetic field to the nanoscale for enhancing light-matter interaction, which are in great demand in SERS [98]. At present, as a powerful ultra-sensitive analysis technology, SERS has a wide range of applications in biological detection and environmental monitoring. In general, an ideal SERS substrate should have uniform and rich "hot spots" to increase Raman intensity and ensure signal repeatability. Fortunately, colloidal lithography may be an effective method to solve this problem. In Fig. 8, Hong and colleagues [99] showed a new technology for trapping "hot spots" by combining SERS with plasma (Fig. 8a). Through plasma capture, they were able to collect gold nanoparticles (GNPs) in the middle of the initially manufactured nano-bowtie structure (Fig. 8b), in which the laser was excited, and the result showed a hot spot formed in the area. At the same time, our group [100] monitored the catalytic reaction using the same silver bowtie nanoantenna arrays. The ordered nanostructures with graphene coating indicate that the catalytic reaction occurs on the chemical inertness of monolayer graphenecoated silver nanostructures (Fig. 9).

Fig. 10 presents the high surface sensitivity and fingerprint information based on SERS for sensing applications [101]. Studies have shown that severely hazardous heavy metal mercury ions can be enriched in the human body through the food chain and other pathways, but it is difficult to detect them using traditional methods. In this case, a colloidal sphere was used to prepare a sandwich structure with a large area of uniform sub-nano gaps, which has extremely high surface sensitivity and fingerprint information about chemical structures, and is used to detect $\mathrm{Hg}^{2+}$ in water through the principle of complementary pairing of biological bases. In addition, the SERS substrate can also be used to detect sandy soils contaminated with $\mathrm{Hg}^{2+}$, and shows excellent performance. Sandwich structures prepared based on colloidal crystals have great potential for detecting toxic metal ions and environmental pollutants because of their high repeatability, large area, uniformity, low cost, easy preparation, and high sensitivity. After that, our group further designed a new volume-enhanced Raman scattering (VERS) substrate with a body "hot spots", as shown in Fig. 11. The substrate consists of an array of micronsized bowls with nano-cone holes at the bottom, and the diameter of the nano-cone pores can be adjusted by the virus' size. These hot spots are composed of hexagonally arranged hollow nano-cones located at the bottom of the micro-bowls. The VERS substrate was prepared in steps as displayed in Fig. 11a [59]. The corresponding SEM images can be seen in Fig. 11b. These VERS substrates 


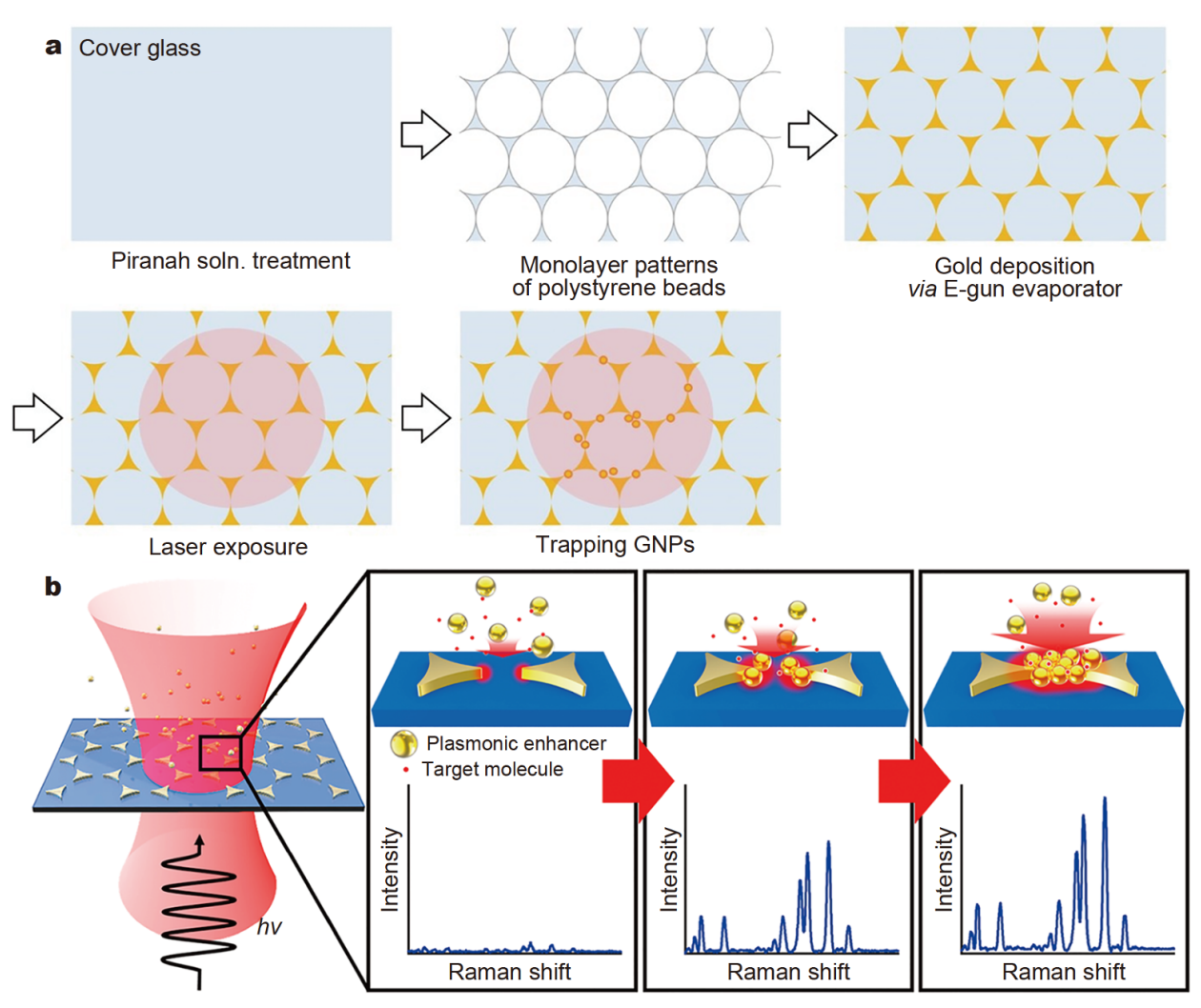

Figure 8 Triangle metallic nanoparticle arrays and metal film on nanospheres (MFON) for SERS based on colloidal lithography. (a) Schematic diagram of the fabrication method for the auto-enhanced Raman spectroscopy (AERS) substrate. (b) Auto-formed SERS substrate by trapping GNPs into the middle of nano-bowtie structures via plasmonic trapping. Reproduced with permission from Ref. [99]. Copyright 2016, American Chemical Society.

allowed for large interfacial area between the virus and the inner surface of the hollow nano-cone, thus increasing the area of the virus that could contribute to the Raman signals (Fig. 11c), also created volume hot spots, and allowed for Raman information collection of the parts of the virus far away from the surface hot spots. The reproducibility of the Raman signals and the detection reliability of single viruses were significantly improved. SERS substrates based on colloidal crystals are of great significance in disease diagnosis and biomedicine.

Recently, inspired by various 2D SERS substrates based on the formation of colloidal crystals, and incorporating the excellent sound absorption performance of natural beehives, we have developed a 3D gold-coated titanium dioxide macro-porous inversed opal (MIO) structure with a template-based sol-gel method, which could perfectly coordinate the interaction of the laser, sample and SERS substrate with engineered "slow light effect" and thus showed outstanding SERS performance [81]. Our design has great potential for making in vitro cancer diagnosis or monitoring as simple as diagnostic monitoring for common diseases. A spin-coating method was used to prepare multilayer polystyrene pellet polystyrene opal templates, as exhibited in Fig. 11d. The sol solution used to prepare titanium dioxide was infiltrated into the interstitials of the polystyrene sphere templates. After annealing to remove the polystyrene spheres and solidifying the framework, we obtained the MIO structures, as shown in Fig. 11e. The corresponding SERS performance is shown in Fig.11f, indicating that the method has a strong universality in cancer diagnosis.

\section{Antireflection}

Microstructures with rich periodicity abound in nature. By studying the relationship between periodic structure and function in natural environments, humans have been inspired to design many devices with excellent performance. Antireflective surfaces (ARS) are a periodic array, which can suppress reflection loss in a large range of wavelengths and increase light transmission at the interface [102-104]. The nipple arrays of nocturnal insects have been discovered as typical antireflective structures $[105,106]$. In practical applications, the ARSs are of significant importance in the area of optical and electro- 

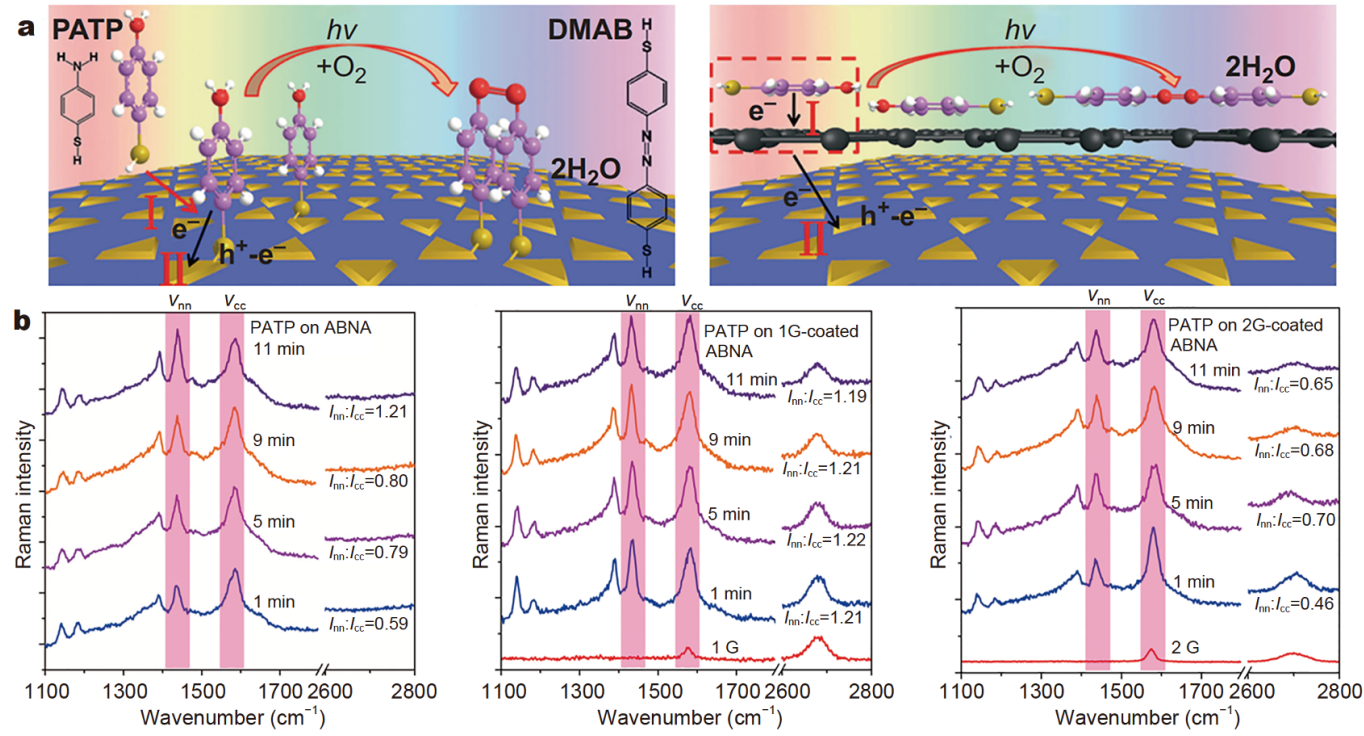

Figure 9 (a) Schematic view of the structure of $p$-aminothiophenol (PATP) and the formation process of dimethylaminobenzaldehyde (DMAB) by the conventional plasmon-driven reaction and the graphene-assisted, plasmon-driven reaction. (b) In situ SERS of PATP on Ag bowtie nanoantenna arrays (ABNA), 1G-coated ABNA, 2G-coated ABNA. Reproduced with permission from Ref. [100]. Copyright 2015, Springer Nature Publishing.

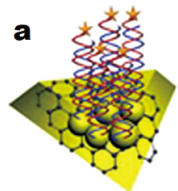

TAMRA-CGT TGT T TG CT T TGC

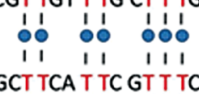

MSH : DNA «W: DNA2 O: Au NPs $\gg:$ Graphene $\rightarrow$ : Au bowtie nanoantenna arrays

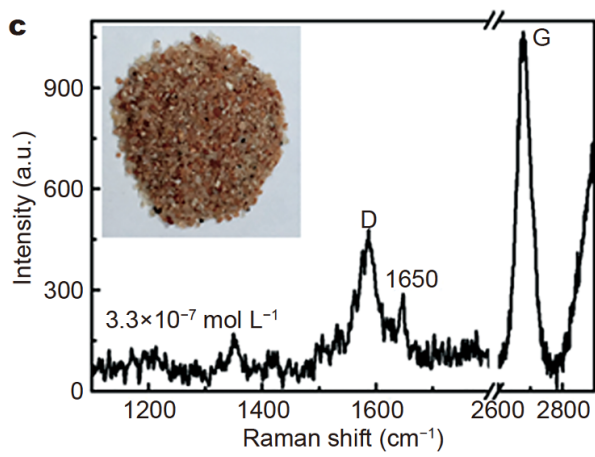

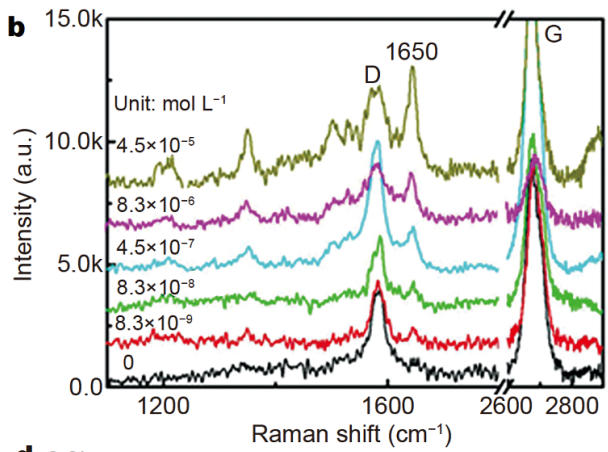

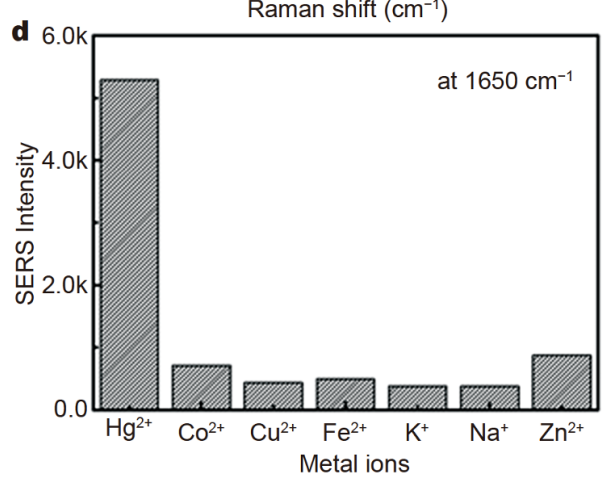

Figure 10 Noble metal nanoarray SERS substrates. (a) Schematic illustration of detection of $\mathrm{Hg}^{2+}$. (b) SERS spectra of different $\mathrm{Hg}^{2+}$ concentrations. (c) SERS detection of $\mathrm{Hg}^{2+}$ released from contaminated sandy soil; the inset is the optical image of sandy soil. (d) Comparison with the other metal ions. Reproduced with permission from Ref. [101]. Copyright 2017, Wiley-VCH.

optical devices, and extensive efforts have been made to overcome the major obstacles of optoelectronic devices, which can improve the antireflection properties. In this regard, MCC-based nanostructures are an ideal candidate for the high-performance ARSs.

To date, there are two main methods for preparing 

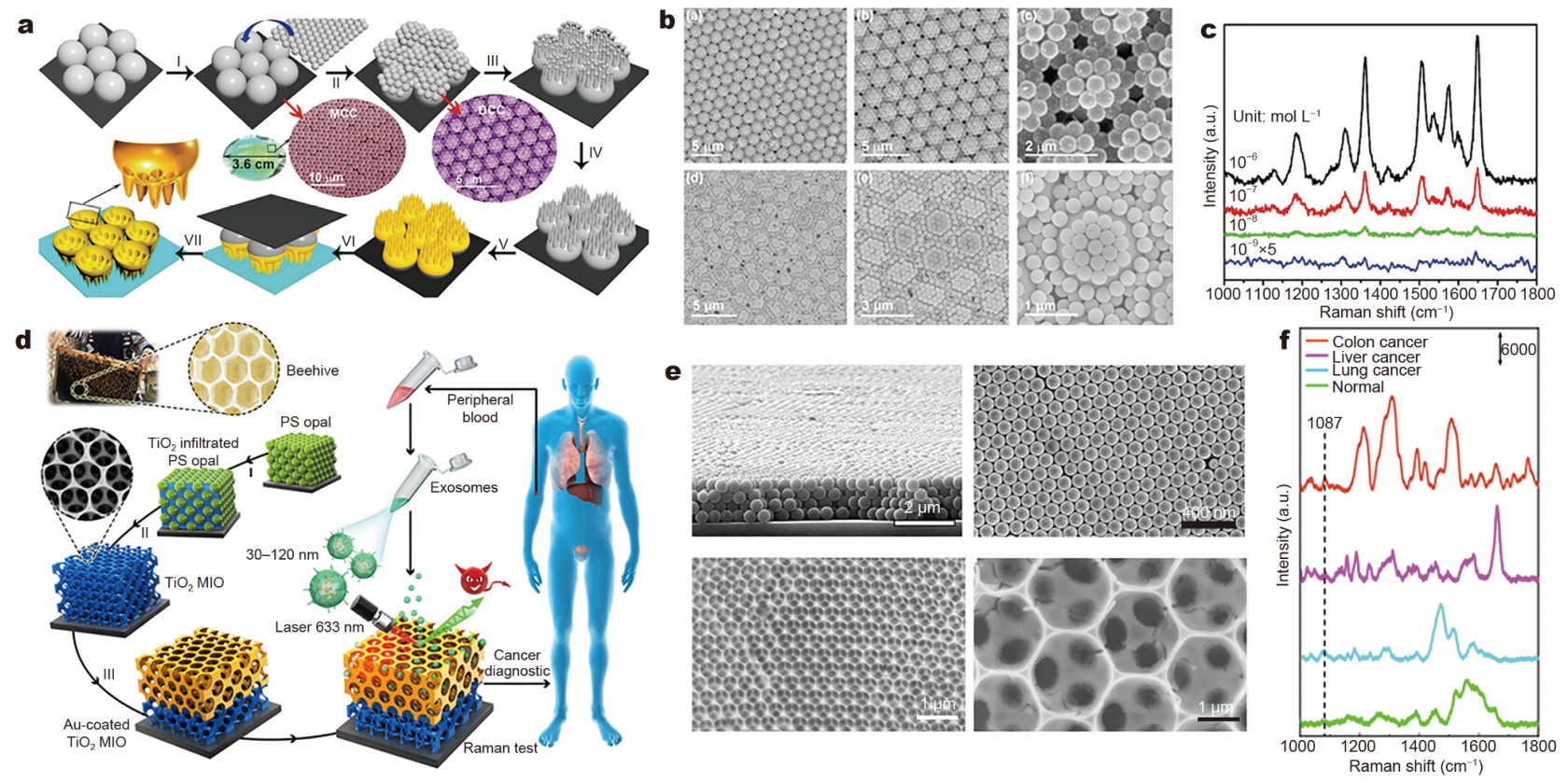

Figure 11 Concept of SERS detection of viruses and exosomes. (a) Fabrication process of the gold hollow nanocones at the bottom of microbowl (HNCMB) structures. (b) Characterization of the plasma-etched DCC template and the HNCMB structures. (c) Detection of viruses using different sensing substrates. Reproduced with permission from Ref. [59]. Copyright 2019, Wiley-VCH. (d) Detection process and design inspiration of Aucoated titanium dioxide MIO SERS probe. (e) SEM images of the substrate in (d). (f) SERS spectra of the exosomes separated from plasma of different cancer patients and normal individuals. Reproduced with permission from Ref. [81]. Copyright 2020, American Chemical Society.

high-efficiency ARSs: one is to apply an anti-reflective coating film to the surface, and the other is to apply a sub-wavelength bionic moth eye structure to the surface. Haesung and co-workers [107] proposed a new method to combine the antireflective coating with the moth-eye structure to make the antireflective spectral range of the nanostructures smaller than the lattice constant without the need for complicated processes to make sub-300 nm feature sizes, as shown in Fig. 12. Yang and co-workers [51] were inspired and utilized DCC templates in conjunction with site-specific electrochemical growth to create soccer ball-like microscale granule structures, which were compatible to various materials including metals, metal oxides, and conductive polymers (Fig. 13). These small-molecule coatings can exhibit strong omnidirectional anti-reflection performance in the range of 250 to $2000 \mathrm{~nm}$, which is comparable to that of the most advanced anti-reflection coatings. An arbitrary MCC template is picked from below using an arbitrary substrate. The substrate can be a smooth, flexible, curved or rough surface. In short, many high-performance ARSs are made with MCC templates, but their performance has not been fully optimized. Manufacturing high-performance and economical ARS remains a challenge, and this ARS will find more applications in solar cells, shielding coatings and many other fields.

\section{Photonic crystals}

Photonic crystals (PCs) are a new class of composite materials patterned with a periodicity in dielectric constant that can create a range of forbidden frequencies called a photonic bandgap $[108,109]$, for which they have been widely used in the field of detection, mainly through the unique photonic band gap-induced structural color, reflectance, and fluorescence enhancement, giving the platform the rich optical information required. The hierarchical periodic structure prepared with the help of MCCs has superior optical properties, providing a means to control and manipulate light, and are periodic arrays of dielectric scatter in homogeneous dielectric matrices. Recently, Qin and colleagues [110] explored the angledependent structural color of PCs (Fig. 14), which provided a wealth of optical information and produced rainbow-colored chips, enabling easy identification of multiple analytes. The film exhibits structural colors in consecutive spectra, which are attributed to the longrange ordered arrangement of latex particles. The singlephoton crystal matrix chip can differentially enhance the 

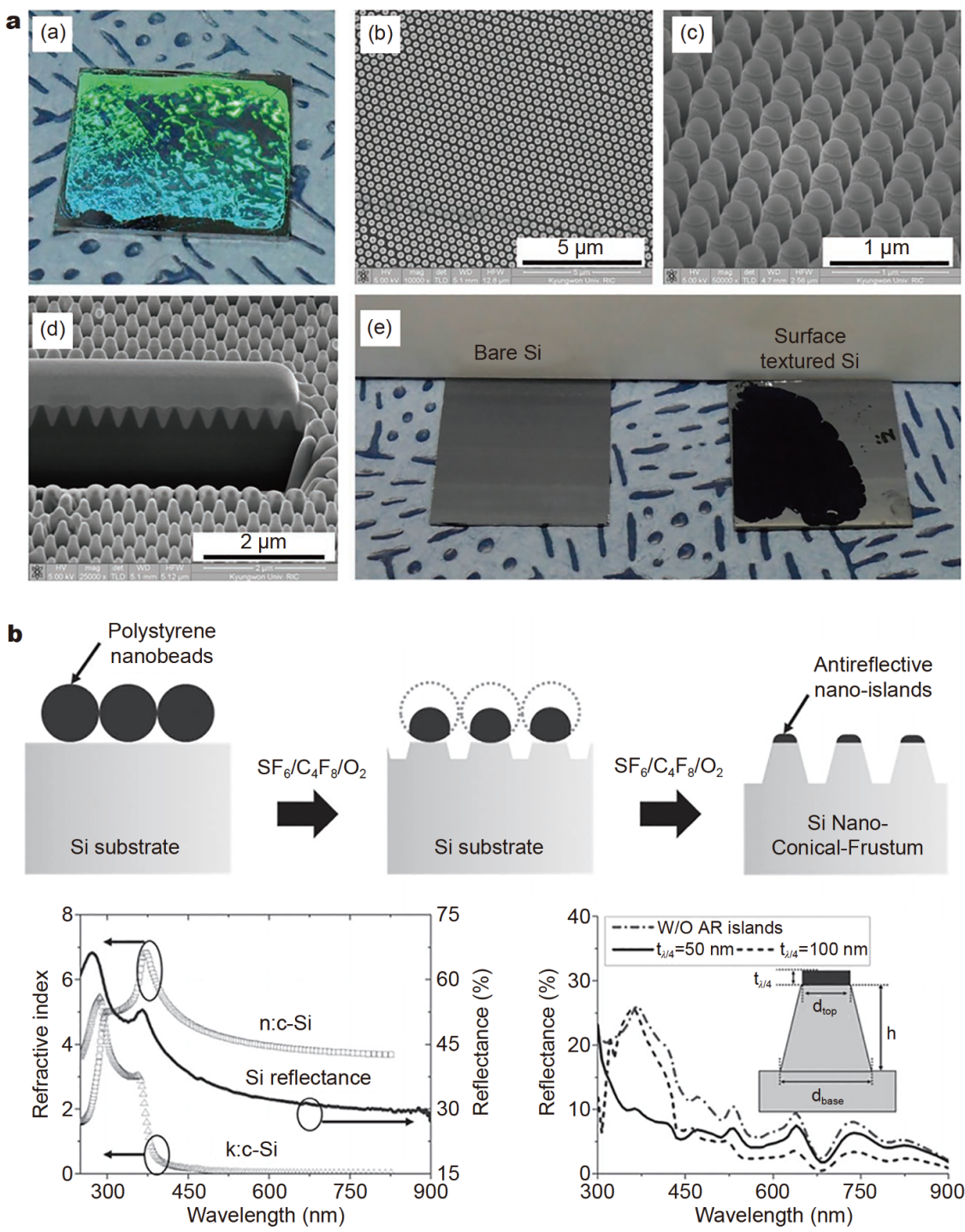

Figure 12 (a) Optical photograph and SEM images of 2D polystyrene sphere bead crystals on Si substrate. (b) Schematic diagram shows the detailed fabrication process of antireflective nano-islands in silicon arrays. Reproduced with permission from Ref. [107]. Copyright 2019, Wiley-VCH.

fluorescence signal in a wide spectral range through a simple multi-angle analysis method, thereby providing rich sensing information for efficient multiple analysis.

\section{Photo-electrochemical application}

Water splitting using photo-electrochemical junction is one of the most promising technologies for a sustainable energy supply and was first reported in 1972 [111]. Photocatalysis plays a very important role in energy conversion and environmental protection. A key challenge in this field is to manufacture high-surface-area nanostructured photoelectrodes with ideal performance properties, such as wide absorption range, high carrier mobility, high stability and environmental friendliness. Morphological engineering provides hope for overcoming this challenge. The hierarchical periodic structure prepared with the help of monomolecular colloidal crystals has enhanced photocatalytic performance, so the synthesis of nanostructured photocatalysts is an important approach. Fig. 15 presents a simple two-step method, including interface nanolithography based on MCCs and hydrothermal growth for the construction of a novel 3D hierarchical $\mathrm{TiO}_{2}$ nanorod@nanobowl (NR@NB), and the unmodified array photoelectrode yields a photocurrent density of $1.24 \mathrm{~mA} \mathrm{~cm}^{-2}$ at $1.23 \mathrm{~V}$ with respect to the reversible hydrogen electrode [112]. 

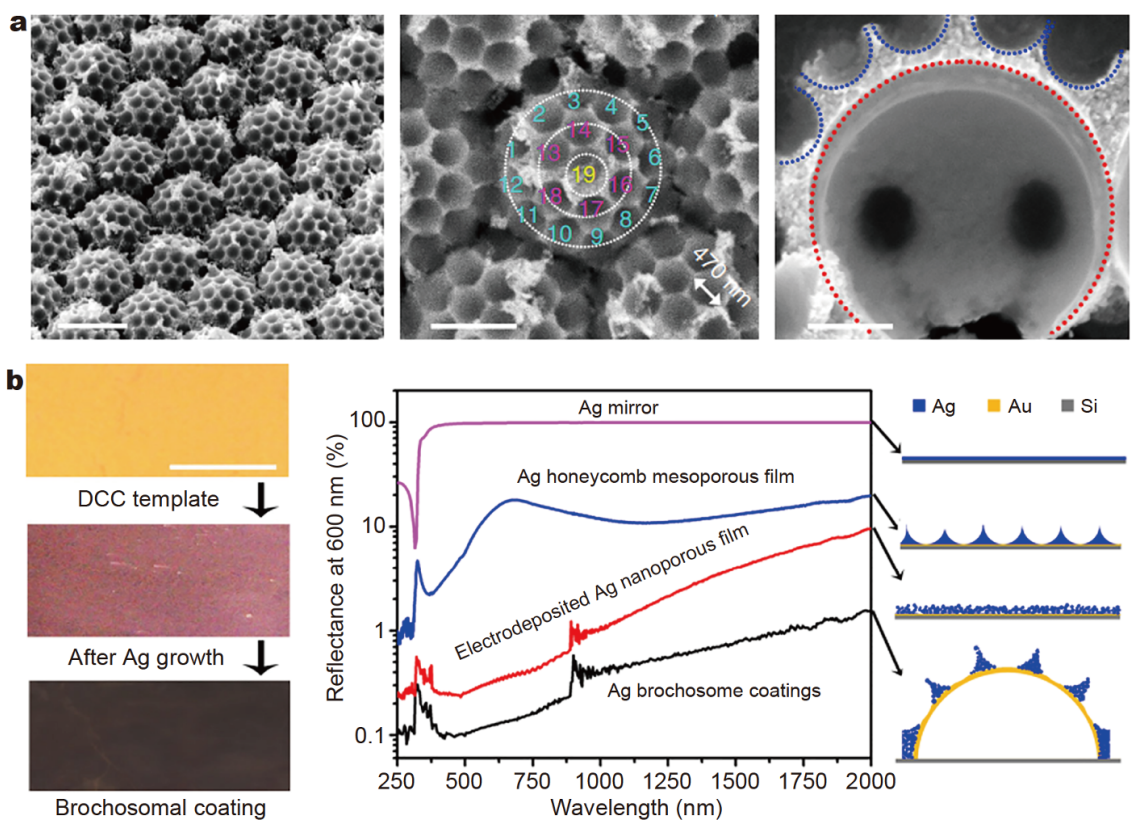

Figure 13 (a) Structural control of binary colloidal crystals. (b) Antireflection performance of the synthetic binary colloidal crystals. Reproduced with permission from Ref. [51]. Copyright 2017, Springer Nature Publishing.
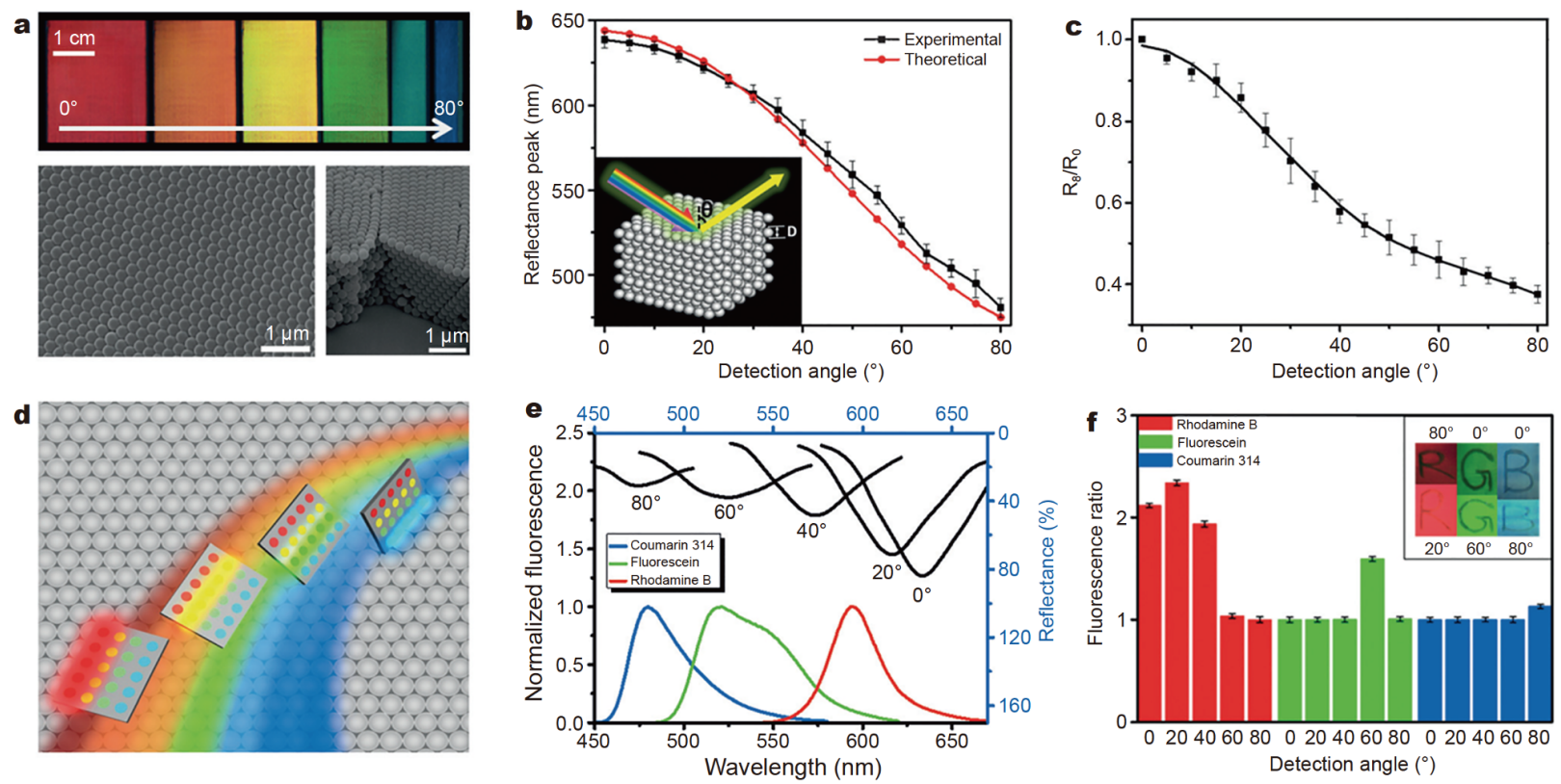

Figure 14 (a) Photograph of the photonic crystals' matrix film with different angles and the corresponding SEM images. (b) Changes in the reflection intensity upon increasing the detection angles. (c) Experimental and theoretical values of the reflection peaks of the PC matrix film with increasing detection angles. (d) Schematic illustration of the rainbow-color photonic crystal chip. (e) Fluorescence spectra of the dyes and stop bands of the photonic crystal matrix film at different detection angles. (f) Changes in the fluorescence of dyed PC matrix films upon increasing the detection angles. Reproduced with permission from Ref. [110]. Copyright 2016, Wiley-VCH.

Ren and co-workers [113] also fabricated a novel 3D hierarchical $\mathrm{TiO}_{2}$ urchin assembled with a hollow $\mathrm{TiO}_{2}$ sphere core and a radial $\mathrm{TiO}_{2} \mathrm{NR}$ shell via a facile colloid template route combined with atomic layer deposition 


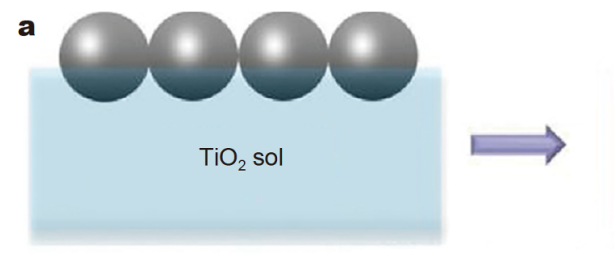

$\mathrm{TiO}_{2}$ deposition
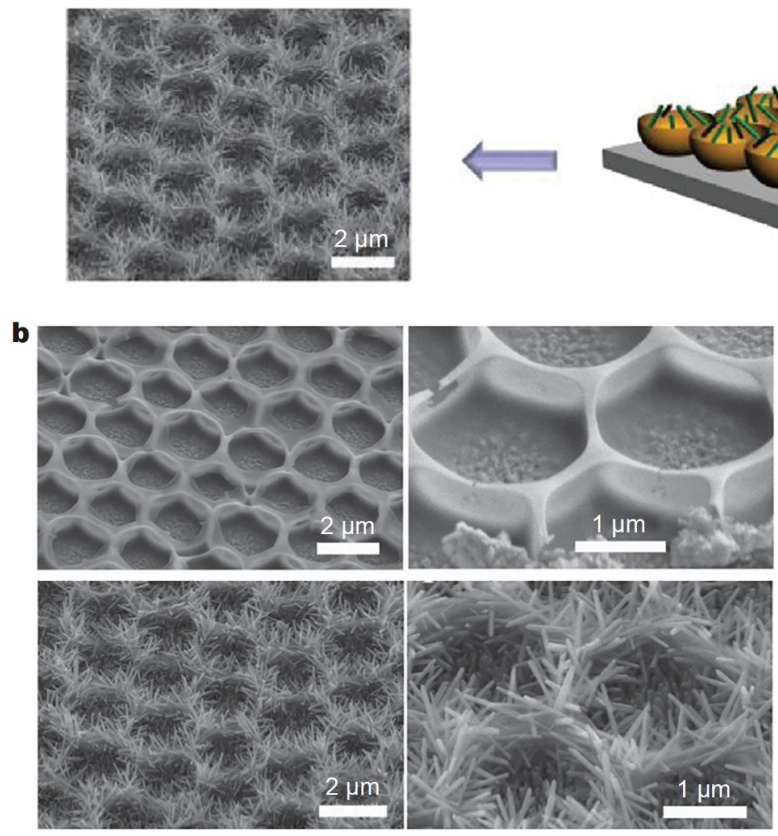

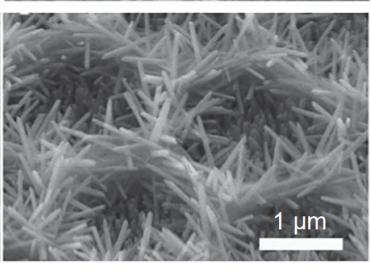

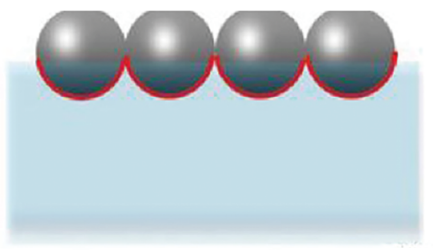

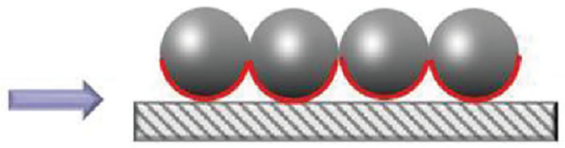

Transfer onto FTO

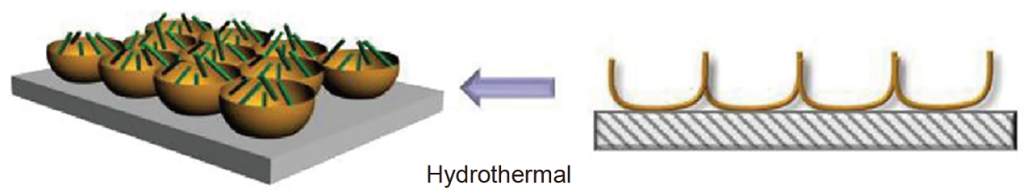

C
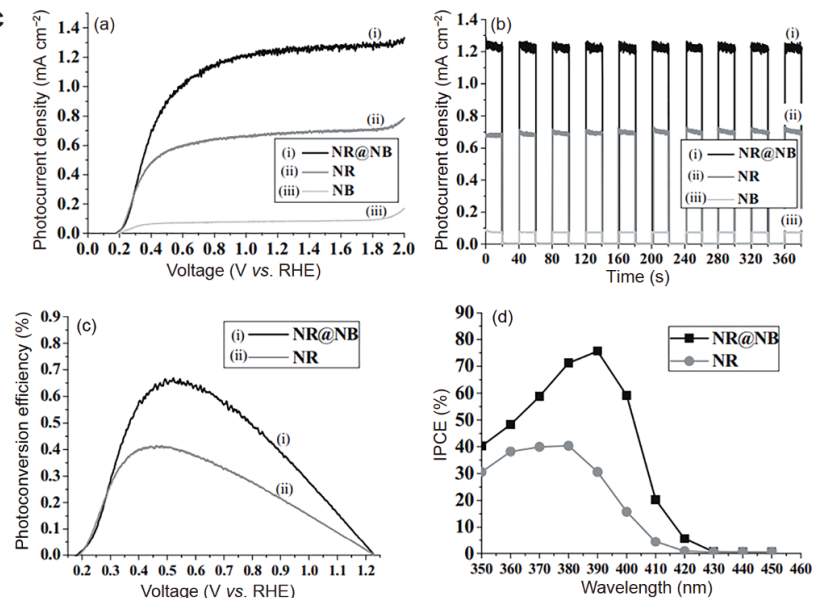

Figure 15 (a) Schematic illustration of the preparation process of $\mathrm{TiO}_{2} \mathrm{NR@NB}$. (b) SEM images of TiO 2 NB arrays and TiO $\mathrm{NR}_{2} @ \mathrm{NB}$ arrays

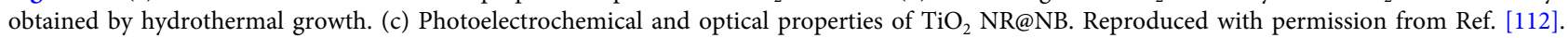
Copyright 2016, Wiley-VCH.

and a hydrothermal growth process. A greatly improved photoelectrochemical water splitting performance was obtained from the structured nanocolumn array of amorphous $\mathrm{TiO}_{2}$ in contrast to the $\mathrm{TiO}_{2}$ microspheres due to the improved light harvesting and charge collection efficiency, which may be ascribed to the ordered hierarchical structures composed of radiation shaped nano-branches emanating from a center point on the polystyrene spheres [114,115].

\section{Practical utilizations for enhanced electronic device domains}

In addition to the above applications, the $2 \mathrm{D}$ patterned nanostructures obtained with colloidal crystals also have great applications in many other fields, such as electronic device applications [116,117]. A narrow-necked periodic graphene nano-grid was prepared by using a single molecular layer colloidal crystal-assisted etching method, and the resulting structure had good electrical conductivity and a high switching ratio of 10 [118]. Based on the self-assembly of MCC, our group [119] reported a simple and low-cost method to fabricate wafer-scale uniform and flexible nanotip array substrates via polystyrene sphere. We have exploited this nanotip array to fabricate high-performance single conductive filament resistive switching memory. As a result, the tailored Ag/ $\mathrm{HfO}_{2} / \mathrm{Au}$ nanotip resistive random-access memory devices on polyethylene terephthalate substrates showed good flexibility, as demonstrated in Fig. 16, a large switching window over $10^{8}$, an ultralow off-state current of $10^{-12} \mathrm{~A}$, and low and uniform programming voltages. This device can also be prepared in other systems to optimize device performance. Additionally, polystyrene spheres (diameter: $700 \mathrm{~nm}$ ) spontaneously formed a monolayer uniform film by surface assembly on water, then they were transferred onto the $\mathrm{Si} / \mathrm{SiO}_{2}$ substrate, as 

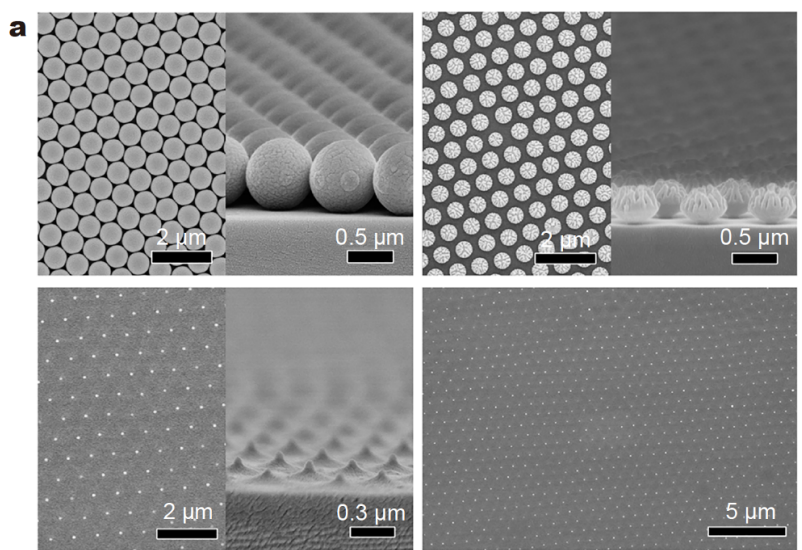
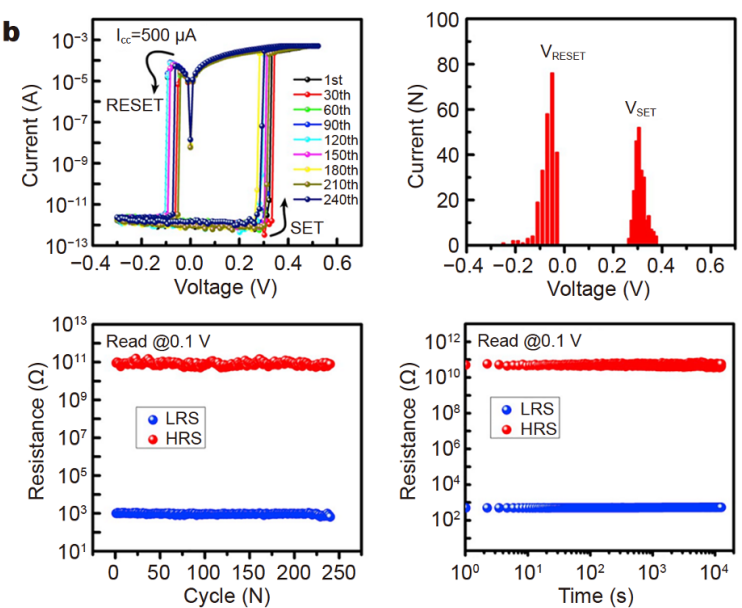

Figure 16 (a) SEM images of the polystyrene spheres and nanotip arrays on a silicon wafer before and after oxygen plasma etching. (b) Switching performance of Au nanotip-based resistive random access memory (RRAM) devices. Reproduced with permission from Ref. [119]. Copyright 2017, Elsevier Ltd.

a

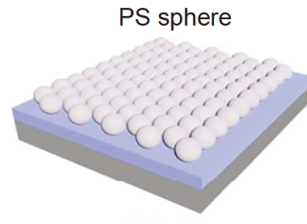

$\mathrm{SiO}_{2} / \mathrm{Si}$
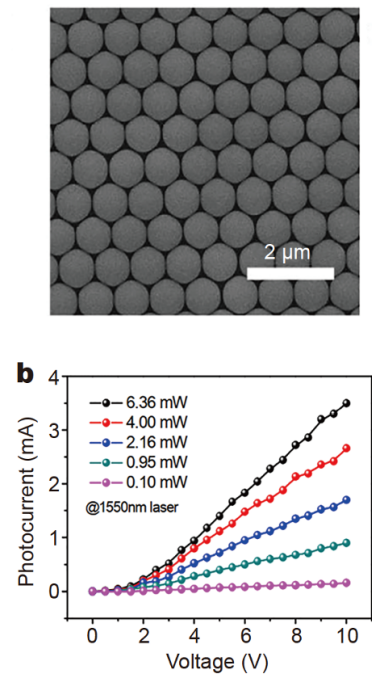

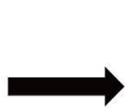

Au depositing

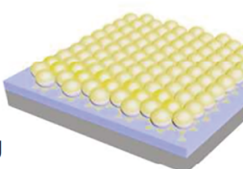

Sphere removing
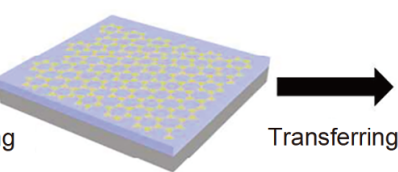

Transferring
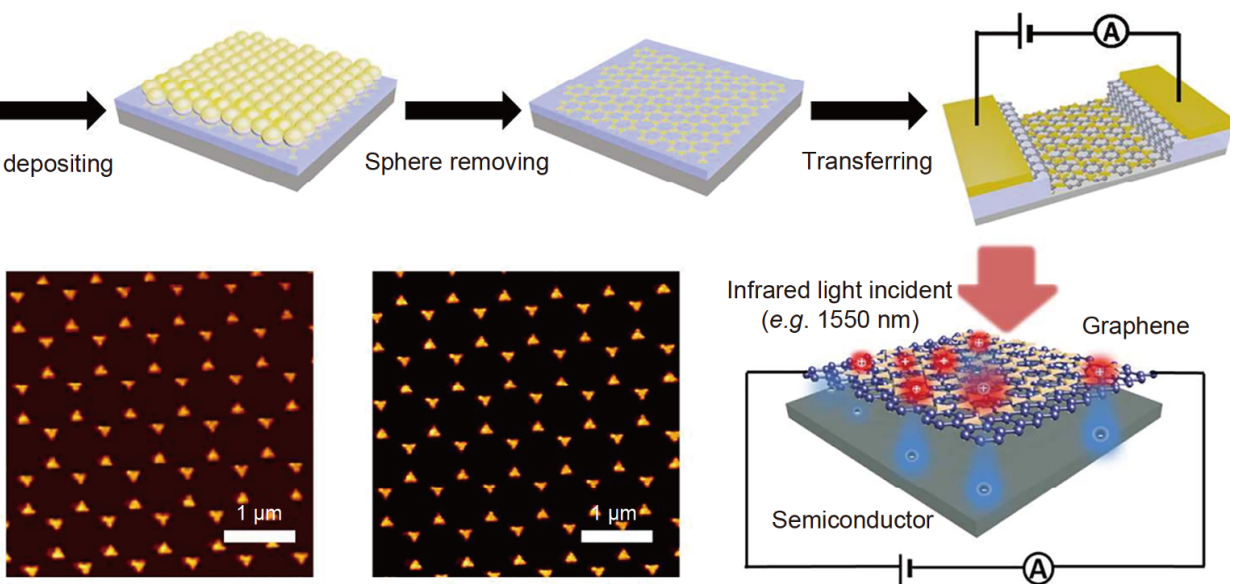

Infrared light incident
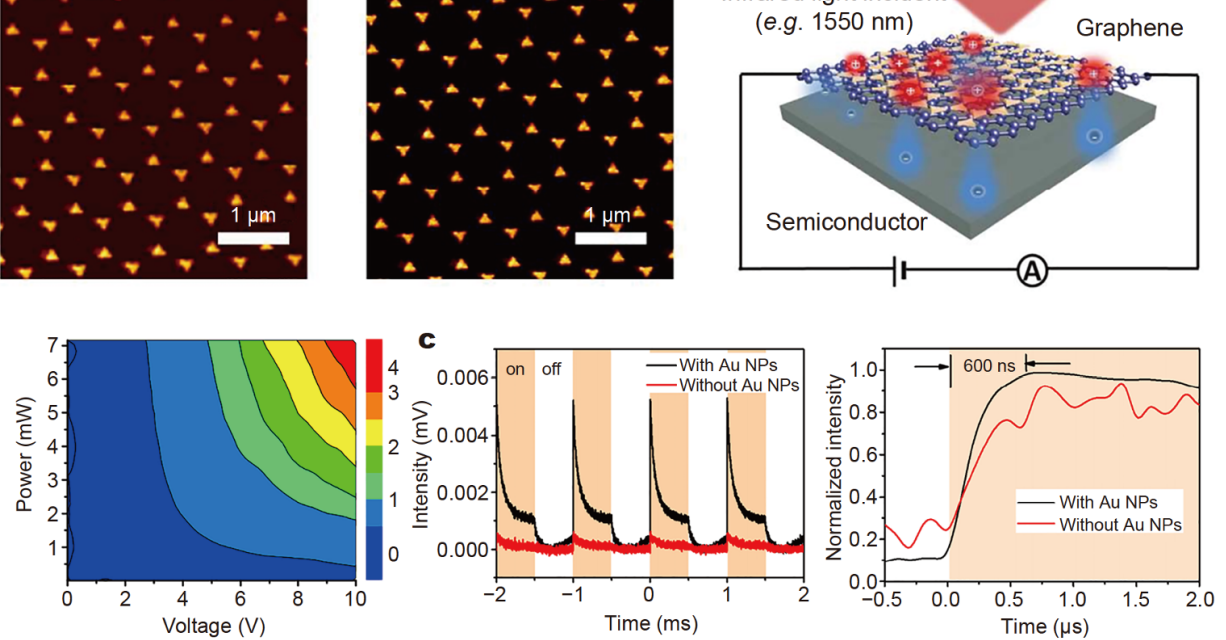

Figure 17 (a) Fabrication of the Au NPs array using monolayer polystyrene spheres as a mask and transferring the Au NPs array to graphene SWIR photodetector and the corresponding SEM images of the monolayer polystyrene spheres on $\mathrm{SiO}_{2}$ substrate obtained by self-assembly. (b) Photocurrent and photoresponsivity measurement of the plasmon resonance enhanced photodetectors. (c) Characterizations of the transient response of graphene SWIR photodetector. Reproduced with permission from Ref. [120]. Copyright 2017, American Chemical Society.

shown in Fig. 17. By using this method, Chen and coworkers [120] fabricated the plasmonic nanostructures coupled graphene short-wave infrared (SWIR) photodetector by nanosphere lithography, which demonstrated as an ultrahigh responsivity and fast photo-response graphene SWIR photodetector. In this way, high-performance and promise for the development of mid/far infrared optoelectronic applications can be realized. 


\section{SUMMARY AND OUTLOOK}

Ordered nanostructures have attracted much attention due to their unique surface properties and broad application prospects in many important scientific and technical fields. The spherical structure of nanospheres has been widely used in the preparation of nanostructures of various shapes, such as triangular metal nanoparticles, circular nanoparticles. With nano-etching technology and metal deposition technology, more complex nanostructures can be obtained now, which will greatly enrich the new functions of nanostructure arrays.

The modification of colloidal crystal spheres is a costeffective and large-scale surface patterning method, and it is also a simple and easy one-step solution for constructing 3D manufacturing. In the colloidal crystal-assisted nanofabrication process, the choice of control method is very important for the formation of the structure. By appropriately changing the experimental conditions, the material, shape, feature size, and period of the resulting nanostructure array can be adjusted. It has been shown that patterned nanostructures based on colloidal crystals show huge potential applications in many important fields, especially in optical aspects, such as photonics, plasma, SERS, antireflection, photonic crystals, photocatalysis, and electronic devices, and this is the focus of this review. Despite great achievements in colloidal sphere self-assembly, subsequent preparation and application of nanostructures have achieved, many specific problems still remain. First, self-assembled single-layer colloidal crystal structures are prone to defects, and these defects will lead to corresponding functional degradation of the following nanostructures. Therefore, the defect-free nature of these self-assembled colloids should be further achieved. Second, due to the inherent limitations of colloidal materials, it is hard to meet industrial requirements. In order to meet the different needs of other technical fields, colloidal materials with different sizes, surface and mechanical properties should be developed. Finally, the relationship between application performances and the structural parameters of nanostructures remains unclear. Considerable effort is required to study the pattern-related properties and application performance in order to rationally design patterned nanostructures with optimized structural parameters for specific applications.

It is believed that with the development of modern nano-manufacturing technology, more and more complex nano-structure arrays will be prepared. This will facilitate large-scale use in photovoltaics, photonics, energy storage, catalysis, and other fields. Based on the self- assembly of these colloidal spheres, as well as all the reviewed functions and promising features of this technology, we hope that $2 \mathrm{D}$ colloidal crystals and nanostructure arrays will bring new functions and promising applications in the near future.

\section{Received 19 January 2020; accepted 1 March 2020; published online 15 April 2020}

1 Kravets VG, Schedin F, Jalil R, et al. Surface hydrogenation and optics of a graphene sheet transferred onto a plasmonic nanoarray. J Phys Chem C, 2012, 116: 3882-3887

2 Ye T, Dai Z, Mei F, et al. Synthesis and optical properties of gold nanorods with controllable morphology. J Phys-Condens Matter, 2016, 28: 434002

3 Boukai AI, Bunimovich Y, Tahir-Kheli J, et al. Silicon nanowires as efficient thermoelectric materials. Nature, 2008, 451: 168-171

4 Vayssieres L. Growth of arrayed nanorods and nanowires of $\mathrm{ZnO}$ from aqueous solutions. Adv Mater, 2003, 5: 464-466

5 Bertholdo R, Assis MC, Hammer P, et al. Controlled growth of anodic aluminium oxide films with hexagonal array of nanometer-sized pores filled with textured copper nanowires. J Eur Ceramic Soc, 2010, 30: 181-186

6 Li JF, Zhang YJ, Ding SY, et al. Core-shell nanoparticle-enhanced Raman spectroscopy. Chem Rev, 2017, 117: 5002-5069

7 Zankovych S, Hoffmann T, Seekamp J, et al. Nanoimprint lithography: challenges and prospects. Nanotechnology, 2001, 12: 91-95

8 Qin D, Xia Y, Whitesides GM. Soft lithography for micro- and nanoscale patterning. Nat Protoc, 2010, 5: 491-502

9 Wei X, Luan L, Zhao Z, et al. Nanofabricated ultraflexible electrode arrays for high-density intracortical recording. Adv Sci, 2018, 5: 1700625

10 Herman A, Trompoukis C, Depauw V, et al. Influence of the pattern shape on the efficiency of front-side periodically patterned ultrathin crystalline silicon solar cells. J Appl Phys, 2012, 112: 113107

11 Zhu X, Wang W, Yan W, et al. Plasmon-phonon coupling in large-area graphene dot and antidot arrays fabricated by nanosphere lithography. Nano Lett, 2014, 14: 2907-2913

12 Zhang XA, Elek J, Chang $\mathrm{CH}$. Three-dimensional nanolithography using light scattering from colloidal particles. ACS Nano, 2013, 7: 6212-6218

13 Lei Y, Yang $\mathrm{S}$, Wu M, et al. Surface patterning using templates: concept, properties and device applications. Chem Soc Rev, 2010, 40: $1247-1258$

14 Lin H, Xiu F, Fang M, et al. Rational design of inverted nanopencil arrays for cost-effective, broadband, and omnidirectional light harvesting. ACS Nano, 2014, 8: 3752-3760

15 Geissler M, Xia Y. Patterning: Principles and some new developments. Adv Mater, 2004, 16: 1249-1269

16 Ye X, Qi L. Two-dimensionally patterned nanostructures based on monolayer colloidal crystals: Controllable fabrication, assembly, and applications. Nano Today, 2011, 6: 608-631

17 Thorkelsson K, Bai P, Xu T. Self-assembly and applications of anisotropic nanomaterials: A review. Nano Today, 2015, 10: 48-66

18 Lei Y, Cai W, Wilde G. Highly ordered nanostructures with tunable size, shape and properties: A new way to surface nanopatterning using ultra-thin alumina masks. Prog Mater Sci, 2007, 
52: 465-539

19 Liang X, Dong R, Ho JC. Self-assembly of colloidal spheres toward fabrication of hierarchical and periodic nanostructures for technological applications. Adv Mater Technol, 2019, 4: 1800541

20 Wang Y, Zhang M, Lai Y, et al. Advanced colloidal lithography: From patterning to applications. Nano Today, 2018, 22: 36-61

21 Yang SM, Jang SG, Choi DG, et al. Nanomachining by colloidal lithography. Small, 2006, 2: 458-475

22 Gates B D, Xu Q, Stewart M, et al. New approaches to nanofabrication: Molding, printing, and other techniques. Chem Rev, 2005, 105: 1171-1196

23 Maury P, Reinhoudt D, Huskens J. Assembly of nanoparticles on patterned surfaces by noncovalent interactions. Curr Opin Colloid Interface Sci., 2008, 13: 74-80

24 Sun J, Li Y, Dong H, et al. Fabrication and light-transmission properties of monolayer square symmetric colloidal crystals via controlled convective self-assembly on 1D grooves. Adv Mater, 2008, 20: 123-128

25 Khanh NN, Yoon KB. Facile organization of colloidal particles into large, perfect one- and two-dimensional arrays by dry manual assembly on patterned substrates. J Am Chem Soc, 2008, 131: 14228-14230

26 Fischer UC, Zingsheim HP. Submicroscopic pattern replication with visible light. J Vacuum Sci Tech, 1981, 19: 881-885

27 Koh SJ. Strategies for controlled placement of nanoscale building blocks. Nanoscale Res Lett, 2007, 2: 519-545

28 Marlow F, Muldarisnur F, Sharifi P, et al. Opals: Status and prospects. Angew Chem Int Ed, 2009, 48: 6212-6233

29 Dziomkina NV, Vancso GJ. Colloidal crystal assembly on topologically patterned templates. Soft Matter, 2005, 1: 265

30 Fan Z, Razavi H, Do JW, et al. Three-dimensional nanopillararray photovoltaics on low-cost and flexible substrates. Nat Mater, 2009, 8: 648-653

31 Lee J, Hua B, Park S, et al. Tailoring surface plasmons of highdensity gold nanostar assemblies on metal films for surface-enhanced Raman spectroscopy. Nanoscale, 2013, 6: 616-623

32 Mubeen S, Zhang S, Kim N, et al. Plasmonic properties of gold nanoparticles separated from a gold mirror by an ultrathin oxide. Nano Lett, 2012, 12: 2088-2094

33 Guo LJ. Nanoimprint lithography: methods and material requirements. Adv Mater, 2007, 19: 495-513

34 Yang R, Terabe K, Liu G, et al. On-demand nanodevice with electrical and neuromorphic multifunction realized by local ion migration. ACS Nano, 2012, 6: 9515-9521

35 Wu R, Ma Y, Pan J, et al. Efficient capture, rapid killing and ultrasensitive detection of bacteria by a nano-decorated multifunctional electrode sensor. Biosens Bioelectron, 2018, 101: 52-59 Denkov N, Velev O, Kralchevski P, et al. Mechanism of formation of two-dimensional crystals from latex particles on substrates. Langmuir, 1992, 8: 3183-3190

37 Juttukonda V, Paddock RL, Raymond JE, et al. Facile synthesis of tin oxide nanoparticles stabilized by dendritic polymers. J Am Chem Soc, 2006, 128: 420-421

38 Li X, Iocozzia J, Chen Y, et al. From precision synthesis of block copolymers to properties and applications of nanoparticles. Angew Chem Int Ed, 2018, 57: 2046-2070

39 Zhao YS, Fu H, Peng A, et al. Construction and optoelectronic properties of organic one-dimensional nanostructures. Acc Chem Res, 2010, 43: 409-418

40 Gao P, He J, Zhou S, et al. Large-area nanosphere self-assembly by a micro-propulsive injection method for high throughput periodic surface nanotexturing. Nano Lett, 2015, 15: 4591-4598

41 Retsch M, Zhou Z, Rivera S, et al. Fabrication of large-area, transferable colloidal monolayers utilizing self-assembly at the air/water interface. Macromol Chem Phys, 2009, 210: 230-241

42 Shinotsuka K, Kajita Y, Hongo K, et al. Crystal perfection of particle monolayer at the air-water interface. Langmuir, 2015, 31: 11452-11457

43 Guo D, Zheng X, Wang X, et al. Formation of multicomponent size-sorted assembly patterns by tunable templated dewetting. Angew Chem Int Ed, 2018, 57: 16126-16130

44 Guo D, Li Y, Zheng X, et al. Programmed coassembly of onedimensional binary superstructures by liquid soft confinement. J Am Chem Soc, 2018, 140: 18-21

45 Hong L, Cacciuto A, Luijten E, et al. Clusters of charged Janus spheres. Nano Lett, 2006, 6: 2510-2514

46 Schüth F, Schmidt W. Microporous and mesoporous materials. Adv Mater, 2002, 14: 629-638

47 Meng Y, Gu D, Zhang F, et al. A family of highly ordered mesoporous polymer resin and carbon structures from organic-organic self-assembly. Chem Mater, 2006, 18: 4447-4464

48 Zhang G, Wang D, Möhwald H. Decoration of microspheres with gold nanodots-Giving colloidal spheres valences. Angew Chem Int Ed, 2005, 44: 7767-7770

49 Zheng J, Dai Z, Mei F, et al. Micro-nanosized nontraditional evaporated structures based on closely packed monolayer binary colloidal crystals and their fine structure enhanced properties. J Phys Chem C, 2014, 118: 20521-20528

50 Zhang X, Xiao X, Dai Z, et al. Ultrasensitive SERS performance in 3D "sunflower-like" nanoarrays decorated with Ag nanoparticles. Nanoscale, 2017, 9: 3114-3120

51 Yang S, Sun N, Stogin BB, et al. Ultra-antireflective synthetic brochosomes. Nat Commun, 2017, 8: 1285

52 Chen S, Yang Z, Meng L, et al. Electromagnetic enhancement in shell-isolated nanoparticle-enhanced Raman scattering from gold flat surfaces. J Phys Chem C, 2015, 119: 5246-5251

$53 \mathrm{Li} \mathrm{X}$. Metal assisted chemical etching for high aspect ratio nanostructures: A review of characteristics and applications in photovoltaics. Curr Opin Solid State Mater Sci, 2012, 16: 71-81

54 Valsesia A, Colpo P, Meziani T, et al. Immobilization of antibodies on biosensing devices by nanoarrayed self-assembled monolayers. Langmuir, 2006, 22: 1763-1767

55 Valsesia A, Mannelli I, Colpo P, et al. Protein nanopatterns for improved immunodetection sensitivity. Anal Chem, 2008, 80: 7336-7340

56 Pearton SJ, Norton DP. Dry etching of electronic oxides, polymers, and semiconductors. Plasma Process Polym, 2004, 2: 16-37

57 Cottat M, Lidgi-Guigui N, Tijunelyte I, et al. Soft UV nanoimprint lithography-designed highly sensitive substrates for SERS detection. Nanoscale Res Lett, 2014, 9: 623-629

58 Esposito M, Tasco V, Todisco F, et al. Three dimensional chiral metamaterial nanospirals in the visible range by vertically compensated focused ion beam induced-deposition. Adv Opt Mater, 2014, 2: 154-161

59 Zhang X, Zhang X, Luo C, et al. Volume-enhanced Raman scattering detection of viruses. Small, 2019, 15: 1805516

$60 \mathrm{Li} \mathrm{X}, \mathrm{Hu} \mathrm{H}, \mathrm{Li} \mathrm{D}$, et al. Ordered array of gold semishells on $\mathrm{TiO}_{2}$ spheres: An ultrasensitive and recyclable SERS substrate. ACS Appl Mater Interfaces, 2012, 4: 2180-2185

61 Fuhrmann B, Leipner HS, Höche HR, et al. Ordered arrays of 
silicon nanowires produced by nanosphere lithography and molecular beam epitaxy. Nano Lett, 2005, 5: 2524-2527

62 Jeong JR, Kim S, Kim SH, et al. Fabrication of hexagonal lattice $\mathrm{Co} / \mathrm{Pd}$ multilayer nanodot arrays using colloidal lithography. Small, 2007, 3: 1529-1533

63 Teng F, Li N, Xu D, et al. Precise regulation of tilt angle of $\mathrm{Si}$ nanostructures via metal-assisted chemical etching. Nanoscale, 2017, 9: 449-453

64 Huang Z, Fang H, Zhu J. Fabrication of silicon nanowire arrays with controlled diameter, length, and density. Adv Mater, 2007, 19: $744-748$

65 Lin D, Wu Z, Li S, et al. Large-area Au-nanoparticle-functionalized Si nanorod arrays for spatially uniform surface-enhanced Raman spectroscopy. ACS Nano, 2017, 11: 1478-1487

66 Xu X, Yang Q, Wattanatorn N, et al. Multiple-patterning nanosphere lithography for fabricating periodic three-dimensional hierarchical nanostructures. ACS Nano, 2017, 11: 10384-10391

67 Alhmoud H, Delalat B, Elnathan R, et al. Porous silicon nanodiscs for targeted drug delivery. Adv Funct Mater, 2015, 25: 1137-1145

68 George SM. Atomic layer deposition: An overview. Chem Rev, 2010, 110: 111-131

69 Peng L, Fang Z, Zhu Y, et al. Holey 2D nanomaterials for electrochemical energy storage. Adv Energy Mater, 2017, 8: 1702179

70 Chen C, Ding T, Qi Z, et al. Fabrication and characterization of vertically aligned $\mathrm{ZnO}$ nanorod arrays via inverted monolayer colloidal crystals mask. Electron Mater Lett, 2018, 14: 467-473

71 Xu Y, Sheng K, Li C, et al. Self-assembled graphene hydrogel via a one-step hydrothermal process. ACS Nano, 2010, 4: 4324-4330

72 Kälblein D, Weitz RT, Böttcher HJ, et al. Top-gate $\mathrm{ZnO}$ nanowire transistors and integrated circuits with ultrathin self-assembled monolayer gate dielectric. Nano Lett, 2015, 11: 5309-5315

73 Wang X, Summers CJ, Wang ZL. Large-scale hexagonal-patterned growth of aligned $\mathrm{ZnO}$ nanorods for nano-optoelectronics and nanosensor arrays. Nano Lett, 2004, 4: 423-426

74 Chen K, Thang DD, Ishii S, et al. Selective patterned growth of $\mathrm{ZnO}$ nanowires/nanosheets and their photoluminescence properties. Opt Mater Express, 2015, 5: 353

75 Zhu C, Meng G, Zheng P, et al. A hierarchically ordered array of silver-nanorod bundles for surface-enhanced Raman scattering detection of phenolic pollutants. Adv Mater, 2016, 28: 4871-4876

76 Wang XD, Graugnard E, King JS, et al. Large-scale fabrication of ordered nanobowl arrays. Nano Lett, 2004, 4: 2223-2226

77 Asoh H, Sakamoto S, Ono S. Metal patterning on silicon surface by site-selective electroless deposition through colloidal crystal templating. J Colloid Interface Sci, 2007, 316: 547-552

78 Zhang H, Zhou F, Liu M, et al. Spherical nanoparticle arrays with tunable nanogaps and their hydrophobicity enhanced rapid SERS detection by localized concentration of droplet evaporation. Adv Mater Interfaces, 2015, 2: 1500031

79 Valsesia A, Lisboa P, Colpo P, et al. Fabrication of polypyrrolebased nanoelectrode arrays by colloidal lithography. Anal Chem, 2006, 78: 7588-7591

80 Davis ME. Ordered porous materials for emerging applications. Nature, 2002, 417: 813-821

81 Dong S, Wang Y, Liu Z, et al. Beehive-inspired macroporous SERS probe for cancer detection through capturing and analyzing exosomes in plasma. ACS Appl Mater Interfaces, 2020, 12: 5136-5146

$82 \mathrm{Wu}$ S, Tan X, Lei J, et al. Ga-doped and Pt-loaded porous $\mathrm{TiO}_{2}-$ $\mathrm{SiO}_{2}$ for photocatalytic nonoxidative coupling of methane. J Am Chem Soc, 2019, 141: 6592-6600
83 Shen $\mathrm{K}$, Zhang L, Chen X, et al. Ordered macro-microporous metalorganic framework single crystals. Science, 2018, 359: 206-210

84 Zhang J T, Chao X, Asher S A. Asymmetric free-standing 2-D photonic crystal films and their Janus particles. J Am Chem Soc, 2013, 135: 11397-11401

85 Cui X, Troadec C, Wee ATS, et al. Surface nanostructure formation and atomic-scale templates for nanodevices. ACS Omega, 2018, 3: 3285-3293

86 Hall AS, Friesen SA, Mallouk TE. Wafer-scale fabrication of plasmonic crystals from patterned silicon templates prepared by nanosphere lithography. Nano Lett, 2013, 13: 2623-2627

87 Kim M, Lin M, Son J, et al. Hot-electron-mediated photochemical reactions: principles, recent advances, and challenges. Adv Opt Mater, 2017, 5: 1700004

88 Chen W, Zhang S, Deng Q, et al. Probing of sub-picometer vertical differential resolutions using cavity plasmons. Nat Commun, 2018, 9: 801

89 Pan D, Wei H, Gao L, et al. Strong spin-orbit interaction of light in plasmonic nanostructures and nanocircuits. Phys Rev Lett, 2016, 117: 166803

90 Zhang S, Gu C, Xu H. Single nanoparticle couplers for plasmonic waveguides. Small, 2014, 10: 4264

91 Xie F, Pang JS, Centeno A, et al. Nanoscale control of Ag nanostructures for plasmonic fluorescence enhancement of nearinfrared dyes. Nano Res, 2013, 6: 496-510

92 Homola J. Present and future of surface plasmon resonance biosensors. Anal Bioanal Chem, 2003, 377: 528-539

93 Ding S Y, Yi J, Li J F, et al. Nanostructure-based plasmonenhanced Raman spectroscopy for surface analysis of materials. Nat Rev Mater, 2016, 1: 16021

94 Mulvihill MJ, Ling XY, Henzie J, et al. Anisotropic etching of silver nanoparticles for plasmonic structures capable of singleparticle SERS. J Am Chem Soc, 2010, 132: 268-274

95 Law S, Yu L, Rosenberg A, et al. All-semiconductor plasmonic nanoantennas for infrared sensing. Nano Lett, 2013, 13: 4569-4574

96 Nie S. Probing single molecules and single nanoparticles by surface-enhanced Raman scattering. Science, 1997, 275: 1102-1106

97 Yao J, Le AP, Gray SK, et al. Functional nanostructured plasmonic materials. Adv Mater, 2010, 22: 1102-1110

98 Cho CH, Aspetti CO, Park J, et al. Silicon coupled with plasmon nanocavities generates bright visible hot luminescence. Nat Photon, 2013, 7: 285-289

99 Hong S, Shim O, Kwon $\mathrm{H}$, et al. Autoenhanced Raman spectroscopy via plasmonic trapping for molecular sensing. Anal Chem, 2016, 88: 7633-7638

100 Dai Z, Xiao X, Wu W, et al. Plasmon-driven reaction controlled by the number of graphene layers and localized surface plasmon distribution during optical excitation. Light Sci Appl, 2015, 4: e342

101 Zhang X, Dai Z, Si S, et al. Ultrasensitive SERS substrate integrated with uniform subnanometer scale "hot spots" created by a graphene spacer for the detection of mercury ions. Small, 2017, 13: 1603347

102 Kim JK, Chhajed S, Schubert MF, et al. Light-extraction enhancement of GaInN light-emitting diodes by graded-refractiveindex indium tin oxide anti-reflection contact. Adv Mater, 2008, 20: 801-804

103 Lu Y, Lal A. High-efficiency ordered silicon nano-conical-frustum array solar cells by self-powered parallel electron lithography. Nano Lett, 2010, 10: 4651-4656

104 Huang YF, Chattopadhyay S, Jen YJ, et al. Improved broadband 
and quasi-omnidirectional anti-reflection properties with biomimetic silicon nanostructures. Nat Nanotech, 2007, 2: 770-774

105 Li Y, Zhang J, Yang B. Antireflective surfaces based on biomimetic nanopillared arrays. Nano Today, 2010, 5: 117-127

106 Rakitov R, Gorb SN. Brochosomal coats turn leafhopper (Insecta, Hemiptera, Cicadellidae) integument to superhydrophobic state. Proc R Soc B, 2013, 280: 20122391

107 Park H, Shin D, Kang G, et al. Broadband optical antireflection enhancement by integrating antireflective nanoislands with silicon nanoconical-frustum arrays. Adv Mater, 2011, 23: 5796-5800

108 Mihi A, Zhang C, Braun PV. Transfer of preformed threedimensional photonic crystals onto dye-sensitized solar cells. Angew Chem Int Ed, 2011, 50: 5712-5715

109 Joannopoulos JD, Villeneuve PR, Fan S. Photonic crystals: putting a new twist on light. Nature, 1997, 386: 143-149

110 Qin M, Huang Y, Li Y, et al. A rainbow structural-color chip for multisaccharide recognition. Angew Chem Int Ed, 2016, 55: 6911-6914

111 Fujishima A, Honda K. Electrochemical photolysis of water at a semiconductor electrode. Nature, 1972, 238: 37-38

112 Wang W, Dong J, Ye X, et al. Heterostructured $\mathrm{TiO}_{2}$ nanorod @nanobowl arrays for efficient photoelectrochemical water splitting. Small, 2016, 12: 1469-1478

113 Ren W, Zhang H, Kong D, et al. A three-dimensional hierarchical $\mathrm{TiO}_{2}$ urchin as a photoelectrochemical anode with omnidirectional anti-reflectance properties. Phys Chem Chem Phys, 2014, 16: 22953-22957

114 Wang Z, Huang B, Dai Y, et al. Highly photocatalytic $\mathrm{ZnO} / \mathrm{In}_{2} \mathrm{O}_{3}$ heteronanostructures synthesized by a coprecipitation method. J Phys Chem C, 2014, 113: 4612-4617

115 Zheng L, Zheng Y, Chen $\mathrm{C}$, et al. Network structured $\mathrm{SnO}_{2} / \mathrm{ZnO}$ heterojunction nanocatalyst with high photocatalytic activity. Inorg Chem, 2009, 48: 1819-1825

116 Lee YJ, Ruby DS, Peters DW, et al. ZnO nanostructures as efficient antireflection layers in solar cells. Nano Lett, 2008, 8: 1501-1505

117 Striemer CC, Fauchet PM. Dynamic etching of silicon for broadband antireflection applications. Appl Phys Lett, 2002, 81: 2980-2982

118 Sinitskii A, Tour JM. Patterning graphene through the selfassembled templates: toward periodic two-dimensional graphene nanostructures with semiconductor properties. J Am Chem Soc, 2010, 132: 14730-14732

119 Li W, Song X, Zhao X, et al. Design of wafer-scale uniform Au nanotip array by ion irradiation for enhanced single conductive filament resistive switching. Nano Energy, 2020, 67: 104213

120 Chen Z, Li X, Wang J, et al. Synergistic effects of plasmonics and electron trapping in graphene short-wave infrared photodetectors with ultrahigh responsivity. ACS Nano, 2017, 11: 430-437

Acknowledgements The authors acknowledge the National Key R\&D Program of China (2018YFA0703700), the National Natural Science Foundation of China (11722543, U1867215, 11875211 and U1932134), the Fundamental Research Funds for the Central Universities (2042019kf0312), Suzhou Key Industrial Technology Innovation Project (SYG201828), and Hubei Provincial Natural Science Foundation (2019CFA036).

Author contributions $\mathrm{Xiao} X$ and Wang $\mathrm{Z}$ designed and supervised the project. Liu J and Zhang X prepared the manuscript. Li W and Jiang C revised the manuscript. All authors contributed to the general discussion.
Conflict of interest The authors declare that no conflict of interest.

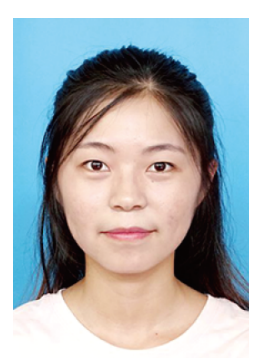

Jing Liu is a PhD student at the School of Physics and Technology, Wuhan University, under Prof. Xiangheng Xiao's supervision. Her research interests are mainly focused on the surface plasmon resonance of novel nanostructures.

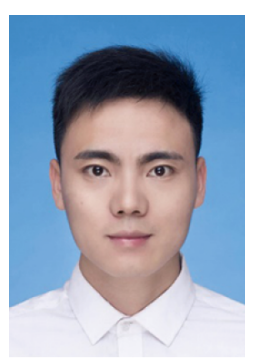

Xingang Zhang received his $\mathrm{PhD}$ from the School of Physics and Technology, Wuhan University, China, in 2019. In the same year, he joined the School of Physics and Electronics, Hunan Normal University, China, as a lecturer. His research interests include the fabrication of SERS platforms and biomedical nanomaterials, and their applications for detection and treatment.

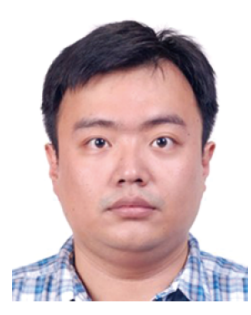

Ziyu Wang is an associate professor in the Institute of Technological Sciences at Wuhan University. He received his $\mathrm{PhD}$ degree from Wuhan University in 2011. From 2011 to 2012, he worked as a postdoctoral in Nanyang Technological University, Singapore. He is focusing on the design synthesis of high-quality nanoplatelets composites, and their thermoelectric properties for potential applications by theoretical analyses and experimental investigations.

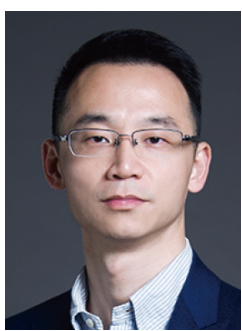

Xiangheng Xiao obtained his BSc in 2001 and $\mathrm{PhD}$ in 2008 from Wuhan University. He is a professor in the School of Physics and Technology, Wuhan University. His research field mainly focuses on surface-enhanced spectroscopy, energy conversion, ion-beam modification of nanoscale materials, and beyond.

\section{胶体球自组装制备周期性结构在光学应用方面的 研究进展}

刘静 $^{1 \dagger}$, 张新刚 $^{3 \dagger}$, 李文庆 ${ }^{1}$, 蒋昌忠 $^{1}$, 王自昱 $2^{*}$, 肖湘衡 ${ }^{1,4^{*}}$

摘要 胶体晶体是由单分散胶体颗粒周期性有序排列而成的一类 新型自组装材料, 在许多领域都具有非常广泛的应用. 基于胶体晶 体自组装的微纳结构具有从几十纳米到几微米可调控的固有周期 性, 这赋予了它们独特的光学性质. 本文主要介绍了近年来胶体球 自组装技术制备周期性图案及其在光学应用方面的研究进展, 具 体包括: (a) 胶体晶体的可控自组装; (b) 通过自组装所获得的胶体 球充当掩模板, 构造更多新颖的功能纳米结构; (c) 图案化纳米结构 在等离激元、抗反射、光子晶体、光催化、电子器件等光学领域 的新特性及应用前景. 最后, 对此领域当前的挑战和未来的前景进 行了展望. 本文旨在通过胶体自组装技术设计更巧妙的周期结构 和探索更广泛的应用. 